\title{
Analyse didactique de l'activité effective des élèves en sciences à l'école primaire
}

Didactic analysis of pupil's real activity in sciences learning in primary school

Joël Bisault et Christine Berzin

\section{(2) OpenEdition}

\section{Journals}

\section{Édition électronique}

URL : https://journals.openedition.org/educationdidactique/510

DOI : $10.4000 /$ educationdidactique. 510

ISBN : 978-2-7535-1621-2

ISSN : 2111-4838

\section{Éditeur}

Presses universitaires de Rennes

\section{Édition imprimée}

Date de publication : 1 juin 2009

Pagination : 77-99

ISBN : 978-2-7535-0873-6

ISSN : 1956-3485

\section{Référence électronique}

Joël Bisault et Christine Berzin, « Analyse didactique de l'activité effective des élèves en sciences à

l'école primaire », Éducation et didactique [En ligne], 3-2 I Juin 2009, mis en ligne le 01 juin 2011 , consulté le 22 juillet 2022. URL : http://journals.openedition.org/educationdidactique/510 ; DOI : https://doi.org/10.4000/educationdidactique.510 


\title{
ANALYSE DIDACTIQUE DE L'ACTIVITÉ EFFECTIVE DES ÉLÉVES EN SCIENCES À L'ÉCOLE PRIMAIRE
}

\author{
Joël Bisault, GRIEST - IUFM de l'Académie d'Amiens / UMR STEF - ENS Cachan \\ Christine Berzin, CLEA, Université de Picardie Jule Verne
}

\begin{abstract}
Résumé : L'activité effective des élèves pendant une séance d'investigation expérimentale à l'école primaire est caractérisée en distinguant quatre types différents d'activité (théorique, observation, technique et «sortie »). Nous observons que l'activité effective est principalement technique ; nous constatons également que les types d'activité principalement visés dans cette séquence didactique (théorique et observation) ne sont obtenus qu'après des interventions répétées du maître. Nous interprétons le glissement observé de l'activité scientifique scolaire vers une activité « ordinaire » par l'ambiguité des tâches prescrites provenant du lien qui peut être fait par les élèves entre les moments considérés ici et d'autres moments scolaires ou non scolaires.
\end{abstract}

Mots - clés : sciences, école primaire, activité effective, expérience, langage

Des moments scolaires de sciences qui articulent des activités expérimentales et langagières

Les programmes actuels ${ }^{1}$ de sciences pour l'école primaire mettent en avant "l'activité des élèves » notamment par le moyen de "l'expérimentation directe $»$. Le lien entre l'enseignement des sciences et la maîtrise de la langue est également mis en avant comme un « aspect essentiel » dans ces textes officiels. Les activités expérimentales et langagières apparaissent ainsi comme des éléments constitutifs d'une démarche scientifique scolaire qui comporte un certain nombre de points communs avec les pratiques de recherche en sciences (Bisault, 2005 c). Cependant, ces deux aspects de l'activité des élèves dans la classe de sciences sont relativement ambigus en termes de visées éducatives car ils peuvent concerner différents registres d'élaboration scientifique. Il faut en effet distinguer le registre du référent empirique et le registre des modèles construits sur ces référents, selon des exigences qui n'ont pas de solution dans le premier registre (Martinand, 2000). Contrairement à d'autres approches didactiques de la modélisation (Tiberghien, Buty, \& Le Maréchal, 2003), nous ne considérons pas que le registre empirique est constitué uniquement d'objets ou de phénomènes - donc de ce qu'on appellerait le réel - mais comporte aussi les connaissances phénoménologiques, phénoménotechniques ou phénoménographiques qui leur sont associées. Dans ce cadre didactique, il n'y a pas opposition ontologique entre modèle et référent mais plutôt une différence d'ordre conjoncturel. Ce qui est référent à un moment donné est une description qui remplace la réalité qu'on ne connaît pas; de la même façon, un modèle peut se substituer à une description antérieure et être incorporé comme phénoménologie dans un nouveau registre empirique (Larcher, 2003). Un des résultats de recherches antérieures sur la modélisation a été d'attirer l'attention sur les difficultés d'élaboration du registre du référent empirique : les objets et phénomènes ne sont pas "donnés » mais ils sont le résultat d'une lecture de la « réalité »- lecture qui n'est pas forcément la même pour les élèves et pour des adultes instruits (Martinand, 2000).

Coquidé (1998) distingue trois modes didactiques de l'activité expérimentale des élèves. Le premier mode est celui de la familiarisation pratique avec des objets et des phénomènes; on peut alors parler d'une « expérienciation». Le second mode est celui de l'investigation empirique; ce qui est en jeu se situe alors dans le processus et la méthode et on peut parler d'une « expérience-objet " (au sens ou c'est l'expérience elle-même qui est l'objet de l'apprentissage). Le troisième mode enfin est celui de l'élaboration théorique; ce qui est en jeu se situe alors au niveau des savoirs et on peut parler alors d'une « expérience-validation». Ce ne sont donc pas les mêmes registres d'élaboration scientifique qui sont en jeu dans chaque cas. En effet, alors que l'expérienciation et l'investigation empirique se situent dans le registre empirique, l'expérience-validation implique nécessairement une mise en relation entre le registre empirique et le registre des modèles. 
De la même façon, les activités langagières peuvent concerner chacun de ces deux registres: Orange, Fourneau, et Bourbigot (2001) distinguent ainsi le débat explicatif se situant au niveau des modèles explicatifs et le débat empirique. Le débat explicatif peut avoir lieu par exemple autour d'écrits de travail proposés par différents groupes d'élèves - chaque écrit de travail constituant l'ébauche d'une explication possible du phénomène étudié. En revanche, le débat reste dans le registre empirique quand la discussion entre élèves porte sur la conformité des productions d'élèves avec les observations réalisées. Il s'agit alors de problèmes de représentation de faits empiriques et non de constructions explicatives.

L'importance des activités langagières en sciences à l'école primaire soulève des questions éducatives qui ne concernent pas seulement l'enseignement des sciences. On peut considérer en particulier que les sciences contribuent au même titre que d'autres domaines à l'apprentissage de la langue (Bisault, 2005 a). De la même façon, l'importance accordée au débat prend tout son sens si on considère que l'éducation civique est le deuxième pôle prioritaire de l'école après la maîtrise de la langue (Bisault \& Le Bourgeois, 2006). Il faut enfin mettre en relation l'importance accordée aux interactions entre pairs avec le renouveau des modèles d'apprentissage socioconstructivistes dans la communauté éducative (Berzin, 2000). Le recours à des activités expérimentales en classe soulève également différents types d'enjeux éducatifs : reproduire en classe certains aspects des pratiques des scientifiques, mettre en place des « activités pratiques », promouvoir le raisonnement et la créativité... (Beaufils \& Larcher, 1999). La combinaison d'activités expérimentales et langagières qui est fortement suggérée dans les textes officiels entraîne donc un croisement de nombreux enjeux éducatifs. Ce croisement peut faciliter le travail des enseignants en permettant des réseaux de pratiques cohérentes (Lebeaume, 2005) ; il peut aussi constituer une difficulté - pour les maîtres comme pour les élèves - en raison du télescopage possible de ces différents enjeux (Bisault, 2005 a). Dans une recherche sur la relation entre le travail de l'enseignant et le travail de l'élève, Bautier dégage deux causes de difficultés particulièrement prégnantes dans le déroulement de la scolarité : «l'identification des objets d'apprentissage et des enjeux cognitifs des tâches et situations », d'une part et le « registre d'activité cognitive et langagière investi par l'élève » d'autre part (Bautier, 2006, p. 112). Ces difficultés nous semblent particulièrement présentes dans l'enseignement des sciences, par exemple dans certains moments scolaires qui articulent des activités expérimentales (notamment manipulatoires) et des activités langagières diverses (discussions entre élèves, écriture de comptes-rendus d'expériences...). Dans cette perspective, nous nous sommes intéressés à des moments scolaires de sciences consacrés à la réalisation d'expériences en petits groupes. Ces moments ont été observés dans une séquence didactique consacrée à l'étude du brouillard au cycle 3 de l'école primaire ${ }^{2}$. Nous avons choisi d'analyser d'une part l'activité effective des élèves pendant ces moments et d'autre part l'influence des interventions du maître sur cette activité effective. Nous développerons plus spécialement le premier versant de cette analyse en nous centrant sur un point de vue de didactique curriculaire. Avec ce point de vue, nous n'analysons pas l'activité des élèves pour étudier ce que chaque élève apprend ${ }^{3}$, ce qui serait essentiel avec un point de vue de didactique des apprentissages. En revanche, nous voulons comprendre comment les enseignants mettent en œuvre les programmes officiels et comment les élèves s'approprient les tâches prescrites par les enseignants et les objets ${ }^{4}$ qui leur sont proposés. L'étude exploratoire que nous présentons ici porte sur un corpus trop réduit pour prétendre répondre même partiellement à ce questionnement. Cependant, comme nous allons le développer ci dessous certaines caractéristiques de cette séquence et certains problèmes qu'elle soulève nous semblent généralisables à un ensemble beaucoup plus large de séquences didactiques de sciences à l'école primaire.

\section{Une séquence didactique qui conduit à des tâches complexes}

La séquence didactique qui fait l'objet de cette analyse présente des caractéristiques assez « classiques » pour les sciences à l'école primaire. Nous en citerons quatre qui sont importantes pour l'analyse des extraits de corpus que nous présentons ici :

- un ancrage sur des objets/phénomènes faisant partie de «l'environnement quotidien » des élèves,

- un recours à l'investigation expérimentale comme moyen d'élaboration cognitive, 
- un usage important du « langage » (oral, écrit, texte, dessin),

- une alternance d'activités (écriture, discussion, réalisation d'expériences...) associées à des modes de regroupement spécifiques (individuel/groupe/ collectif) conduisant à une succession dans une même séance de différentes phases de travail bien contrastées ${ }^{5}$ tant sur le plan des tâches prescrites que sur celui de leur organisation pédagogique ${ }^{6}$.

Trois questions proposées par l'enseignant ont servi de fil conducteur à cette séquence :

Q1 : Qu'est-ce que le brouillard?

Q2 : Comment se forme le brouillard?

Q3 : Peut-on/comment faire du brouillard en classe?

Ces différentes questions nécessitent la mise en relation entre deux registres : celui des modèles et celui du référent empirique. L'étude du brouillard en classe présente a priori des difficultés sur chacun de ces deux registres. Sur le plan empirique, le phénomène physique évoqué (condensation de la vapeur d'eau dans l'air) est difficilement reproductible en classe; de plus, une observation non instrumentée ne permet pas d'accéder à l'échelle des gouttelettes d'eau en suspension dans l'air (trop petites pour être vues à l'œil nu). Sur le plan du modèle, l'explication de la formation du brouillard fait intervenir des notions peu accessibles à l'école primaire (saturation de l'air en vapeur d'eau par exemple) et pose des questions de changement d'échelle ${ }^{7}$ difficilement compréhensibles sans approche quantitative.

Nous présentons ci-dessous le descriptif général de cette séquence avec le détail des deux phases de travail planifiées par l'enseignant qui ont fait l'objet de notre analyse (phases 1 et 2 de la deuxième séance). Avant d'analyser l'activité effective des élèves, nous allons caractériser les tâches prescrites pour chacune de ces deux phases.

\begin{tabular}{|l|l|}
\hline Séance 1 & $\begin{array}{l}\text { Première formulation des réponses aux questions « Qu'est-ce que le brouillard ? » et } \\
\text { «Comment se forme le brouillard? »- conception de protocoles expérimentaux }\end{array}$ \\
\hline Séance 2 & $\begin{array}{l}\text { Phase } 0 \text { : discussion collective des protocoles expérimentaux } \\
\text { Phase } 1: \text { réalisation par groupe d'expériences pour « fabriquer du brouillard » } \\
\text { Phase } 2: \text { écriture par groupe d'un compte-rendu d'expérience }\end{array}$ \\
\hline Séance 3 & $\begin{array}{l}\text { Mise en commun et discussion des résultats expérimentaux } \\
\text { Structuration des connaissances à partir de la lecture de documents }\end{array}$ \\
\hline
\end{tabular}

Document 1 : descriptif général des séances sur le brouillard

Dans la phase 1, l'activité se situe principalement sur le plan expérimental : chaque groupe d'élèves doit réaliser une expérience qui reproduit le mécanisme de formation de brouillard. Cependant, l'organisation de ce travail en petits groupes entraîne nécessairement une activité langagière : les élèves doivent discuter entre eux pour coordonner les actions à effectuer et se mettre d'accord sur les résultats à retenir.

Au contraire, dans la phase 2, l'activité se situe principalement sur le plan langagier : chaque groupe doit en effet rédiger un compte-rendu de l'expérience qu'il vient de réaliser. L'activité expérimentale est donc présente ici au second plan comme contenu discursif des discussions orales entre élèves et de l'écrit qu'ils élaborent en commun.
Ces deux phases correspondent donc à des activités a priori très différentes mais qui contribuent en fait à un même enjeu général : tester les modèles explicatifs élaborés lors de la séance précédente. Il s'agit en effet de mettre en relation des observations qui vont être réalisées en classe et des hypothèses qui ont été formulées auparavant sur le mécanisme de formation du brouillard dans la nature. L'activité expérimentale se situe donc dans le mode didactique de "l'expérience validation » avec une nécessaire mise en relation des données empiriques et des modèles explicatifs. De la même façon, l'activité langagière doit conduire a priori à un débat entre élèves sur le plan empirique comme sur le plan explicatif. Ces deux phases de travail conduisent donc à des tâches complexes qui mettent en jeu deux modalités d'activité complémentaires et deux registres différents d'élaboration scientifique qui doivent être mis en relation. 


\begin{tabular}{|c|c|c|c|}
\hline Phases de travail & Tâche expérimentale & Tâche langagière & Enjeu cognitif \\
\hline $\begin{array}{c}\text { Séance } 2 \text { phase } 1 \\
\text { (réalisation de l'expérience) } \\
\text { En groupes }\end{array}$ & $\begin{array}{l}\text { Réaliser l'expérience } \\
\text { et produire des données } \\
\text { empiriques }\end{array}$ & $\begin{array}{l}\text { Discuter pour coordonner les actions } \\
\text { à effectuer et se mettre d'accord sur les } \\
\text { résultats à retenir }\end{array}$ & \multirow{2}{*}{$\begin{array}{l}\text { Tester les modèles } \\
\text { explicatifs: } \\
\text { mettre en relation les } \\
\text { nouvelles données } \\
\text { empiriques avec les } \\
\text { modèles préalables }\end{array}$} \\
\hline $\begin{array}{c}\text { Séance } 2 \text { phase } 2 \\
\text { (écriture du compte-rendu) } \\
\text { En groupes }\end{array}$ & $\begin{array}{l}\text { Rendre compte } \\
\text { des actions et des } \\
\text { observations }\end{array}$ & $\begin{array}{l}\text { Rédiger collectivement un compte-rendu } \\
\text { d'expérience }\end{array}$ & \\
\hline
\end{tabular}

Document 2 : caractérisation des tâches prescrites

\section{Grille d'analyse de l'activité effective des élèves}

Nous allons préciser maintenant l'activité des élèves que nous avons analysée dans cette recherche ainsi que les indicateurs que nous avons utilisés pour cette séquence didactique. Il est clair que l'activité des élèves évoquée ci-dessus est avant tout une activité intellectuelle qui engage chaque élève à la fois individuellement et au sein d'une organisation collective (petit groupe de travail dans notre cas). Bien entendu, l'activité intellectuelle n'est pas directement observable; on ne peut accéder qu'indirectement et que partiellement aux opérations mentales, par exemple en analysant les productions langagières des élèves. Sur ce plan, l'interprétation des discours doit être faite avec beaucoup de prudence car on ne peut pas penser les rapports entre opérations mentales et indicateurs linguistiques sur le mode de la simple correspondance (Nonnon, 2001). C'est pour cette raison que nous avons utilisé le terme "activité effective » plutôt que le terme « activité réelle ${ }^{8}$ » qui pourrait laisser penser à une totale « transparence » des sujets observés.

Notre projet n'étant pas d'observer ce que chaque élève apprend individuellement mais plutôt de comprendre la dynamique d'ensemble de ces moments scolaires, c'est donc l'activité collective que nous cherchons à analyser. En revanche, c'est au travers d'actions individuelles - en particulier des actions langagières - que nous pouvons avoir accès à cette activité collective. Nous adoptons ici la position de Bronckart qui considère que l'action langagière est la part individuelle d'une activité sociale médiatisée par le langage qui se matérialise par la production d'une entité singulière : le texte ${ }^{9}$ (Bronckart 1996). Nous reprenons donc à notre compte la distinction entre action (située du côté de la personne) et activité (située du côté du collectif organisé) généralement retenue par les didacticiens de différents domaines disciplinaires à la suite des travaux de Léontiev, Bakhtine, Bronckart ou Fillietaz ${ }^{10}$ (Jaubert \& Rebière, 2000; Schubauer-Leoni, Leutenegger, Ligozat, \& Fluckiger, 2007).

Les données utilisées dans notre recherche sont donc les «produits » de ces actions individuelles qui se manifestent tout au long du travail des élèves: des produits langagiers (oraux et écrits) et des gestes constitutifs d'actions sur des objets matériels. Il faudrait rajouter à ces gestes, l'ensemble des gestes symboliques qui peuvent accompagner ces actions ou compléter les interactions verbales. Ces gestes semblent avoir joué un rôle relativement réduit pour cette séquence didactique ${ }^{11}$ et nous ne les avons pas inclus dans notre analyse.

L'essentiel de notre analyse repose sur la distinction entre registre empirique et registre des modèles, mais, comme nous l'avons déjà indiqué, ces deux registres ne sont pas des entités définies a priori mais des registres qui se définissent de façon dynamique en relation mutuelle et dans un contexte donné. Il ne s'agit donc pas d'observer comment les élèvent accèdent à ces deux registres mais plutôt comment ils les construisent ${ }^{12}$. Un premier niveau de construction est déjà disponible au début des phases de travail observées ici : en effet, les affiches produites par les groupes lors de la séance précédente énoncent certains éléments des modèles explicatifs envisagés et proposent des dispositifs permettant de recueillir des observations empiriques. Avec ce que nous venons de dire, il faut être très prudent pour attribuer chaque action observable des élèves à tel ou tel registre. Nous le ferons en fait par rapport à ce que nous estimons être le niveau de construction actuelle du groupe considéré. Par exemple, nous attribuerons au niveau du registre des modèles ce qui déjà été énoncé par les élèves comme un élément explicatif préalable. 
Une autre difficulté est liée à la dimension manipulatoire de l'expérience. Il est clair que l'activité expérimentale envisagée ici implique nécessairement une activité manipulatoire; mais la réciproque n'est pas vraie. Des actions sur des objets ne peuvent contribuer à une activité expérimentale que si elles contribuent, directement ou indirectement, à la production de données empiriques. Il faut donc envisager que des manipulations puissent être totalement déconnectées de l'activité expérimentale voire ne pas se situer réellement dans un registre scientifique. Pour cette raison, nous avons choisi de distinguer deux types d'activité manipulatoire effective : les activités manipulatoires liées clairement aux observations empiriques - comme par exemple dans le cas qui nous intéresse la réalisation de mesures - et les autres activités manipulatoires sans lien évident avec les données empiriques. Cette distinction nous paraît valable non seulement pour les actions sur des objets mais également pour l'ensemble des actions observables. Nous avons utilisé ainsi le terme «observation» pour qualifier une activité liée aux observations empiriques qu'elle se manifeste à l'écrit, à l'oral ou par des actions matérielles. De la même façon nous avons utilisé le terme « technique » pour l'ensemble des activités liées aux aspects techniques de la tâche que cette tâche soit manipulatoire ou langagière. En revanche, nous avons utilisé le qualificatif « théorique » pour désigner les activités se rapportant au registre des modèles; nous avons voulu souligner par ce qualificatif deux caractéristiques des modèles explicatifs en jeu dans cette séquence didactique : leur relative autonomie par rapport aux expériences réalisées et leur aspect spéculatif. Enfin, si nous voulons analyser l'activité effective d'élèves "réels", il est nécessaire d'envisager des activités sans aucun rapport avec la tâche prescrite : nous avons utilisé dans ce cas le terme « sortie ». Nous avons donc envisagé quatre types d'activité effective :

- l'activité théorique (THE) qui se situe obligatoirement dans le registre des modèles,

- l'activité observationnelle (OBS) qui se situe a priori dans le registre empirique,

- l'activité technique (TEC) qui peut se situer dans le registre empirique ou dans un registre non scientifique,
- l'activité de sortie (SOR) quand l'activité sort du registre « scolaire $»$.

Ce sont les activités théorique et d'observation (et leur mise en relation) qui sont principalement visées dans cette séquence mais l'activité technique est un passage obligatoire pour les élèves (comme il l'est dans les pratiques des chercheurs).

Comme nous l'avons déjà indiqué, nous avons voulu analyser l'activité effective à partir des actions individuelles observables qu'elles soient langagières ou matérielles. Nous sommes amenés à envisager trois modalités différentes de réalisation de ces actions en distinguant d'une part action sur des objets et action langagière et d'autre part langage oral et langage écrit :

- $\quad$ une modalité orale (ORA),

- $\quad$ une modalité écrite (ECR),

- $\quad$ une modalité matérielle ${ }^{13}$ (MAT).

Les trois modalités sont parfois difficilement séparables, par exemple, quand un élève commente ce qu'il est en train d'écrire ou quand l'écriture est autant une manipulation d'instruments d'inscription qu'une production de texte. Cependant, ces trois modalités correspondent a priori à des données observables différentes nécessitant des traitements spécifiques: les énoncés oraux doivent être retranscrits à partir d'enregistrements sonores, les productions écrites doivent être recueillies et parfois réécrites pour les rendre plus lisibles, les actions sur les objets doivent être filmées puis décrites. Ces contraintes matérielles nous ont amenés à limiter nos observations à un seul groupe d'élèves afin de pouvoir espérer recueillir le maximum d'éléments reflétant leur activité effective.

Si on considère les différents types d'activité envisageables a priori et les différentes modalités possibles de réalisation, nous sommes amenés à interpréter chaque action individuelle comme relevant d'un des registres d'activité que nous avons définis. Cette interprétation a inévitablement un caractère assez spéculatif et il est sans doute envisageable d'interpréter différemment les données que nous avons recueillies. Nous avons déjà indiqué un critère pour 
déterminer ce qui relève selon nous du registre théorique; les exemples que nous présentons dans la suite permettront de clarifier notre posture d'analyse.

En croisant les 4 types d'activité et les 3 modalités de réalisation des actions, on obtient 12 possibilités ${ }^{14}$ résumées dans le tableau ci-dessous; chacune de ces possibilités est illustrée par un exemple d'action que nous avons observée dans cette séquence. Cette grille de lecture de l'activité effective nous amène à rapprocher des activités extrêmement différentes : ainsi une manipulation d'objets (ex : déballer des glaçons MAT TEC) est placée dans le même registre « technique » qu'une prise en compte que nous avons jugée « technique » d'une tâche verbale (ex : vérifier la présentation et l'orthographe ECR TEC). Ces catégories (et leurs dénominations) sont évidemment discutables et nous sommes conscients du caractère relativement arbitraire de ces distinctions qui ont pour nous une utilité pratique (en tant qu'indicateurs des comportements observables des élèves) plus qu'un fondement théorique; cependant, cette catégorisation est utile pour rapprocher des activités relevant de problèmes didactiques assez voisins. En effet, comme nous l'avons déjà indiqué, une des conditions d'apprentissages est la mise en relation entre tâche immédiate et enjeu cognitif de la tâche; ce problème semble se poser de façon assez voisine quelle que soit la modalité de l'activité (orale, écrite ou matérielle).

\begin{tabular}{|c|c|c|c|c|}
\hline $\begin{array}{c}\text { Modalité } \\
\text { d'action } \\
\text { d'activité }\end{array}$ & Théorique & Observation & Technique & "Sortie» \\
\hline Orale & $\begin{array}{c}\text { ORA THE } \\
\text { Discuter des } \\
\text { interprétations ou } \\
\text { modèles théoriques }\end{array}$ & $\begin{array}{c}\text { ORA OBS } \\
\text { Parler des données } \\
\text { observées }\end{array}$ & $\begin{array}{c}\text { ORA TEC } \\
\text { Discussion sur les } \\
\text { objets manipulés }\end{array}$ & $\begin{array}{c}\text { ORA SOR } \\
\text { Une discussion sans } \\
\text { aucun rapport avec la } \\
\text { séquence }\end{array}$ \\
\hline Écrite & $\begin{array}{l}\text { ECR THE } \\
\text { Produire un texte } \\
\text { explicatif }\end{array}$ & $\begin{array}{c}\text { ECR OBS } \\
\text { Noter des résultats } \\
\text { expérimentaux }\end{array}$ & $\begin{array}{l}\text { ECR TEC } \\
\text { Vérifier la } \\
\text { présentation et } \\
\text { l'orthographe }\end{array}$ & $\begin{array}{c}\text { ECR SOR } \\
\text { Écrire un petit mot doux }\end{array}$ \\
\hline Matérielle & $\begin{array}{c}\text { MAT THE } \\
\text { Pas réalisable }\end{array}$ & $\begin{array}{c}\text { MAT OBS } \\
\text { Faire des mesures }\end{array}$ & $\begin{array}{c}\text { MAT TEC } \\
\text { Déballer des glaçons }\end{array}$ & $\begin{array}{c}\text { MAT SOR } \\
\text { Jouer avec une peluche }\end{array}$ \\
\hline
\end{tabular}

Document 3 : grille d'analyse de l'activité effective des élèves

\section{Analyse de l'activité effective des élèves lors d'un travail en groupes}

\section{Contenu du protocole expérimental utilisé par le groupe}

L'analyse a été réalisée à partir d'une observation portant exclusivement sur un groupe de quatre élèves $^{15}$ : Annie, Dorothée, Marielle et Valérian (identifiés par An, Do, Ma et Va dans le corpus), pendant les deux phases de travail que nous avons décrites ci-dessus. Avant de présenter quelques éléments de cette analyse, nous allons examiner le contenu du «protocole expérimental » rédigé sur une grande affiche par ce groupe à la fin de la séance précédente (séance 1 du document1). La description de ce contenu ${ }^{16}$ est en effet importante pour notre analyse si on considère que ce document fixe le cadre à la fois théorique, observationnel et technique que ce groupe d'élèves a choisi pour guider l'activité qui va suivre dans la deuxième séance qui est analysée ici.

La première partie de l'affiche (reproduite dans le document 4) est un énoncé du «modèle théorique » provisoire élaboré par les élèves lors des premières phases de la séance ${ }^{17}$ pour répondre à la question « comment se forme le brouillard? » : c'est l'influence de la température qui est mise en avant pour expliquer la formation du brouillard. Cette influence est présentée de trois façons successives comme pour en souligner l'importance : d'abord en faisant appel à une expression du langage commun ( «quand il fait frai »), puis de façon plus scientifique en faisant appel à une grandeur quantifiable (« quand la température baisse ») et à une valeur de référence 
(«- 0 ») et enfin en soulignant verbalement l'importance du facteur thermique ( très très froid »). Assez curieusement, le terme « brouillard » est remplacé par « brume » ce qui n'est pas très gênant compte tenu de la similarité de ces phénomènes météorologiques: la distinction entre les deux se limite à un critère de visibilité. Dans la deuxième partie de l'affiche - qui répond à la question « qu'est-ce que le brouillard? »- le phénomène est identifié à la "rosée du matin», elle-même décrite comme une "petite pluie "; cette deuxième partie est une reformulation d'observations empiriques que les élèves ont pu réaliser avant cette séquence didactique. On peut noter que cette formulation indique une compréhension au moins partielle de la nature physique du brouillard (minuscules gouttes d'eau). Ces deux parties de l'affiche séparées par un trait horizontal correspondent dans notre grille d'analyse à deux types différents d'activité effective (ECR THE et ECR OBS).

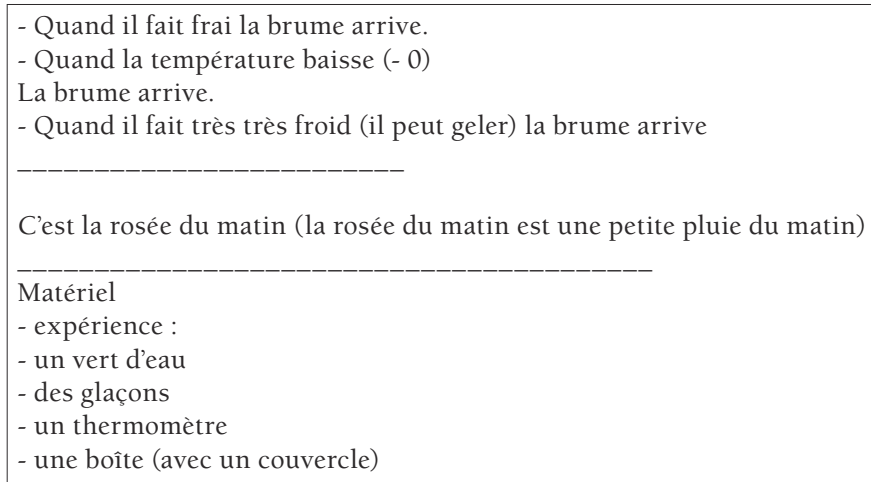

Document 4 : protocole expérimental utilisé par le groupe observé

La dernière partie de l'affiche (également séparée par un trait horizontal) correspond au protocole proprement dit qui se réduit à une liste de matériel. De façon cohérente avec la première partie de l'affiche, la liste de matériel comprend une quantité d'eau et un récipient pour la contenir ( "un vert d'eau»), un moyen de refroidissement (" des glaçons"), un instrument de repérage de la température ( « un thermomètre »), et ce qui peut être compris comme une enceinte de confinement (" une boîte avec un couvercle »). Cette dernière partie comporte donc des aspects techniques qui sont - implicitement au moins - corrélés aux observations à réaliser pour tester le modèle (ECR OBS et ECR TEC). Sans juger de la pertinence scientifique ou technique de ces propositions, nous pouvons remarquer que le texte élaboré par les élèves est globalement conforme à la tâche prescrite par l'enseignant avec une mise en œuvre des différents types d'activité nécessaires à la mise en relation du registre des modèles et du registre empirique. Nous allons maintenant examiner comment ce protocole a été mis en œuvre par les élèves de ce groupe, en présentant d'abord l'architecture générale du corpus étudié et en examinant ensuite quelques extraits représentatifs de ce corpus.

\section{Présentation générale du corpus analysé}

Le recueil du corpus a été réalisé à partir d'un enregistrement vidéo utilisant une caméra positionnée pour observer simultanément les différents élèves du groupe. Tous les échanges verbaux entre élèves ont été retranscrits ainsi que les interventions du maître dans ce groupe ou auprès de l'ensemble de la classe ${ }^{18}$. Cette transcription a été complétée en traduisant verbalement les principales actions matérielles visibles dans l'enregistrement.

À un premier stade de l'analyse nous avons découpé l'ensemble du corpus en unités correspondant à une certaine constance dans les activités mises en ouvre par les élèves. Ces unités sont délimitées par des événements déclencheurs et se caractérisent par le maintien d'un même « environnement de travail » (Schneuwly, Dolz, \& Roveaux, 2006). Nous avons appelé « épisodes » ces unités, en reprenant un terme couramment utilisé dans le découpage d'unités linguistiques observables dans le déroulement réel d'une séance. Ce déroulement réel ne correspond pas forcément à la planification préalable; en effet, l'ordre et la réalisation des 


\section{ANALYSE DidACTIQUe de L'ACTIVIté EFFECTIVE DES ÉLÈVES EN SCIENCES À L'ÉCOLE PRIMAIRE}

\section{Joël Bisault \& Christine Berzin}

épisodes dépendent de l'importance que leur accordent à chaud les participants de l'interaction, c'està-dire non seulement l'enseignant mais surtout les élèves (Bouchard \& Traverso, 2006). Dans notre cas, les événements déclencheurs sont essentiellement liés à l'arrivée du maître dans le groupe étudié - arrivée suivie généralement de nouvelles consignes de travail et dans un des cas de l'introduction d'un nouveau support d'activité. Le découpage que nous avons obtenu coïncide partiellement avec la planification prévue initialement par l'enseignant (cf. document 1). On retrouve ainsi les deux grandes phases correspondant à des tâches prescrites différentes et à des environnements de travail spécifiques (matériel expérimental ou fiche de compte-rendu) mais chacune de ces phases est découpée en unités plus petites résultant de différents moments d'appropriation de ces tâches et d'ajustements de ces tâches par l'enseignant en fonction des réactions des élèves. Le tableau ci-dessous présente le découpage en épisodes que nous avons obtenu ainsi que les principaux éléments observés qui ont permis ce découpage; les moments d'activité dirigée par le maître sont signalés par des lignes grisées.

\begin{tabular}{|c|c|c|c|c|}
\hline Temps & $\begin{array}{l}\text { Intitulé des } \\
\text { épisodes } \\
\text { d'activité } \\
\end{array}$ & Résumé de l'activité des élèves & Evènements déclencheurs & $\begin{array}{l}\text { Environnement } \\
\text { de l'activité }\end{array}$ \\
\hline \multicolumn{5}{|c|}{ Phase 1 : Expérimentation } \\
\hline $\begin{array}{l}15^{\prime} 45-19^{\prime} 55 \\
\quad\left(4^{\prime} 10\right)\end{array}$ & $\begin{array}{l}\text { Episode } 1.1 . \\
\text { «Déballage des } \\
\text { glaçons » }\end{array}$ & $\begin{array}{l}\text { Préparation matérielle de } \\
\text { l'expérience }\end{array}$ & $\begin{array}{l}\text { Mise à disposition du matériel } \\
\text { expérimental }\end{array}$ & $\begin{array}{l}\text { Activité autonome } \\
\text { Utilisation } \\
\text { du matériel } \\
\text { expérimental }\end{array}$ \\
\hline $\begin{array}{l}19,55-20 ' 58 \\
\left(1^{\prime} 03\right)\end{array}$ & $\begin{array}{l}\text { Episode } 1.2 \text {. } \\
\text { Intervention du } \\
\text { maître }\end{array}$ & Discussion sur le protocole & Arrivée du maître & Activité dirigée \\
\hline $\begin{array}{c}20^{\prime} 58-27^{\prime} 39 \\
\quad\left(6^{\prime} 41\right)\end{array}$ & $\begin{array}{l}\text { Episode } 1.3 . \\
\text { Expérimentation } \\
\text { effective }\end{array}$ & Réalisation des observations & Reformulation de la consigne & $\begin{array}{l}\text { Activité autonome } \\
\text { Utilisation } \\
\text { du matériel } \\
\text { expérimental }\end{array}$ \\
\hline $\begin{array}{l}27^{\prime} 39-29^{\prime} 07 \\
\quad\left(1^{\prime} 28\right)\end{array}$ & $\begin{array}{l}\text { Episode } 1.4 . \\
\text { Intervention du } \\
\text { maître }\end{array}$ & $\begin{array}{l}\text { Discussion sur les résultats } \\
\text { puis présentation de la fiche par } \\
\text { le maître }^{19}\end{array}$ & Arrivée du maître & Activité dirigée \\
\hline \multicolumn{5}{|c|}{ Phase 2 : Compte rendu } \\
\hline $\begin{array}{l}29^{\prime} 07-32^{\prime} 30 \\
\left(3^{\prime} 24\right)\end{array}$ & $\begin{array}{l}\text { Episode } 2.1 . \\
\text { «Remplissage } \\
\text { de la fiche }\end{array}$ & $\begin{array}{l}\text { Rédaction parasitée par les } \\
\text { aspects «techniques" de } \\
\text { l'écriture }\end{array}$ & $\begin{array}{l}\text { Changement de support } \\
\text { d'activité }\end{array}$ & $\begin{array}{l}\text { Activité autonome } \\
\text { Utilisation d'une } \\
\text { fiche }\end{array}$ \\
\hline $\begin{array}{l}32^{\prime} 30-33^{\prime} 00 \\
\quad(30 \mathrm{~s})\end{array}$ & $\begin{array}{l}\text { Episode } 2.2 . \\
\text { Intervention du } \\
\text { maître }\end{array}$ & $\begin{array}{l}\text { Discussion s avec le maître sur } \\
\text { le contrat d'écriture }\end{array}$ & Arrivée du maître & Activité dirigée \\
\hline $\begin{array}{c}33^{\prime} 00-35^{\prime} 00 \\
\left(2^{\prime}\right)\end{array}$ & $\begin{array}{l}\text { Episode } 2.3 \text {. } \\
\text { R é d a c t i o n } \\
\text { effective de la } \\
\text { fiche }\end{array}$ & $\begin{array}{l}\text { Rédaction recentrée sur les } \\
\text { observations réalisées }\end{array}$ & Reformulation de la consigne & $\begin{array}{l}\text { Activité autonome } \\
\text { Utilisation d'une } \\
\text { fiche }\end{array}$ \\
\hline $\begin{array}{l}35^{\prime} 00-39^{\prime} 12 \\
\left(4^{\prime} 12\right)\end{array}$ & $\begin{array}{l}\text { Épisode } 2.4 \text {. } \\
\text { Intervention du } \\
\text { maître }\end{array}$ & $\begin{array}{l}\text { Discussion avec le maître } \\
\text { sur la retranscription des } \\
\text { observations }\end{array}$ & Arrivée du maître & Activité dirigée \\
\hline $\begin{array}{l}39 ' 12-41^{\prime} 50 \\
\quad(2 ’ 38)\end{array}$ & $\begin{array}{l}\text { Épisode } 2.5 . \\
\text { Fin de la rédaction } \\
\text { de la fiche }\end{array}$ & $\begin{array}{l}\text { Rédaction recentrée sur les } \\
\text { observations principales }\end{array}$ & Reformulation de la consigne & $\begin{array}{l}\text { Activité autonome } \\
\text { Utilisation d'une } \\
\text { fiche }\end{array}$ \\
\hline
\end{tabular}

Document 5 : découpage du corpus en épisodes 
Le premier épisode de la première phase débute après une intervention collective du maître (phase 0 non représentée dans le tableau) visant à définir la tâche à effectuer et s'achève avec l'arrivée de l'enseignant dans le groupe concerné alors que les élèves ont perdu de vue les investigations à réaliser pour se consacrer exclusivement au déballage des glaçons en déchirant les alvéoles dans lesquelles ils sont contenus. Le troisième épisode fait suite à l'intervention de l'enseignant qui a « recadré » les élèves et ré-orienté leur activité vers un facteur expérimental important : la température de l'eau. Cet épisode est voué à l'expérimentation proprement dite; il s'achève lorsque le maître revient dans le groupe, sollicite les élèves sur les observations réalisées pour les inciter ensuite à passer à l'étape suivante du travail demandé : l'écriture du compte-rendu. La phase d'écriture du compte rendu commence par un épisode assez comparable à celui du « déballage des glaçons » (épisode 2.1.) au cours duquel les élèves se montrent davantage préoccupés par l'orthographe des mots ou par le partage des instruments d'inscription que par la restitution des points essentiels de l'expérimentation. Une nouvelle intervention du maître recentre l'activité des élèves en focalisant notamment leur attention sur les aspects essentiels à faire ressortir. Le travail des élèves qui suit (épisode 2.3.) s'achève par une dernière intervention du maître visant à questionner les résultats de l'expérience. Après quoi, les élèves terminent leur compte-rendu (épisode 2.5.).

\section{Analyse de l'activité effective}

À un deuxième stade de l'analyse, le corpus a été découpé en tranches de trente secondes. L'activité effective des élèves a été analysée pour chacune de ces tranches en prenant en compte les différentes actions langagières et matérielles observées. Nous allons présenter et discuter quelques extraits de ce corpus en indiquant pour chaque extrait, les modalités et types d'activité que nous avons identifiés selon le codage présenté dans notre grille d'analyse ${ }^{20}$.

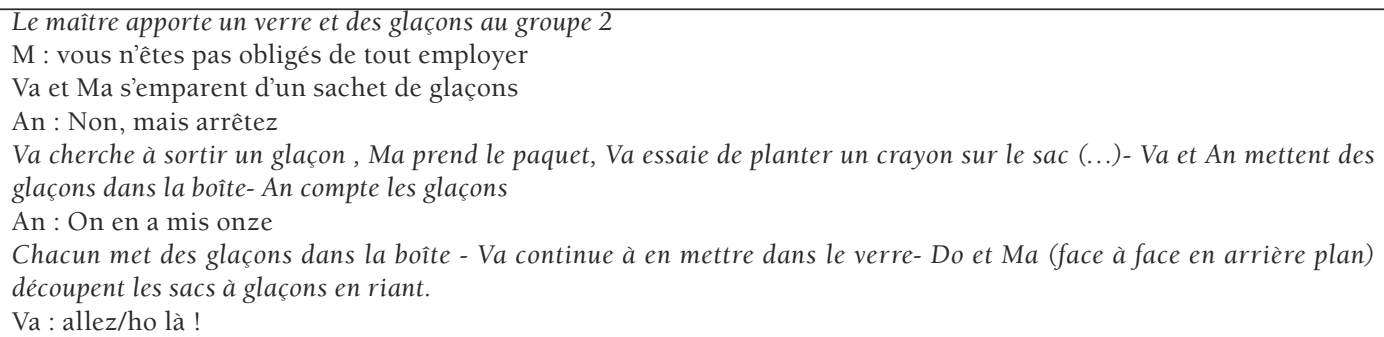

Extrait 1 (épisode 1.1.)

Dans le premier épisode assez long (4 min 10), les élèves se consacrent essentiellement au « déballage des glaçons » en déchirant les sacs à alvéoles en matière plastique; les actions sont assez peu coordonnées. Les actions matérielles comme les discussions orales sont situées dans le type technique, ce que nous avons traduit par (MAT TEC + ORA TEC) dans notre analyse (extrait 1). Ce moment de travail strictement technique s'accompagne de quelques conflits voire d'une sortie totale de la tâche à la fin de l'épisode quand Valérian et Annie se querellent à propos des éclaboussures (extrait 2). L'enseignant (vigilant) intervient peu de temps après pour recentrer l'activité de ce groupe, ce qui fait ressortir dans un premier temps un désaccord technique. L'intervention du maître permet ensuite de recadrer les élèves et d'orienter leur activité vers une dimension plus scientifique en revenant sur un facteur expérimental important : la température de l'eau.

\footnotetext{
An : ben qui m'en met sur mon pull? (en regardant sa manche d'un ton énervé) tu m'énerves (en s'adressant à Va) /oh tu m'en remets sur mon pull ORA SOR

Va: non

M : Alors (elle arrive devant le groupe)/ pourquoi vous voulez absolument les.../ les vider là dedans ?/ c'était fait justement pour.../ quand on le trempe / ça refroidit

Do : Ben / c'est ce que je leur ai dit / ils ont fait n'importe quoi

+ ORA TEC

$\mathrm{M}$ : ben oui mais ça/... (en levant les bras) / vous avez oublié votre expérience pour vous amuser avec les glaçons :

je crois / (...)/ parce que maintenant / l'eau que vous aviez / vous aviez mis de l'eau froide ou de l'eau chaude?
} 
Après ce recadrage, l'activité des élèves guidée par le questionnement du maître revient vers le côté intellectuel de la tâche et sur « l'hypothèse théorique » de l'influence de la température avancée par écrit sur l'affiche du groupe (extrait 3). L'action des élèves est donc directement orientée par les observations à réaliser (MAT OBS) alors que les discussions orales permettent un retour sur les modèles théori- ques préalables (ORA THE). En effet, la discussion ne porte pas sur une observation effective mais sur un élément présent dans le modèle (seuil de température). Le caractère prédictif du modèle se traduit par l'usage du subjonctif « ça fasse ». C'est donc ici par le recours au registre du modèle que les élèves peuvent justifier les activités d'observation qu'ils sont en train de mettre en place.

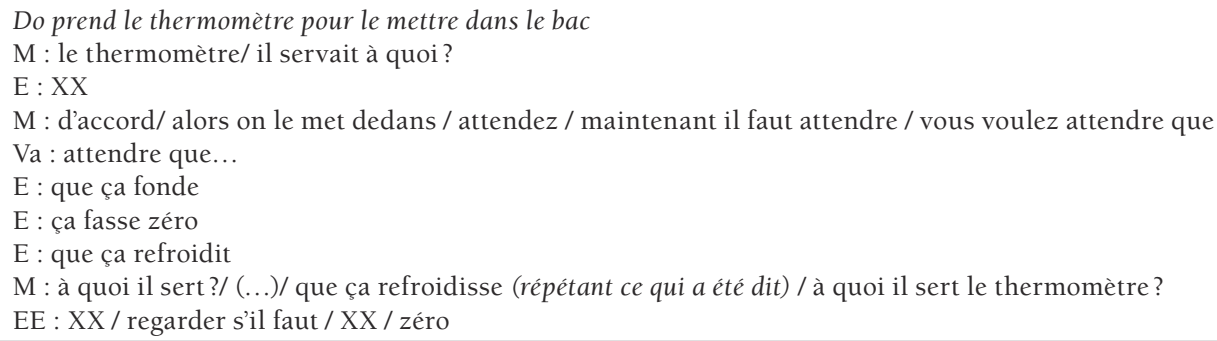

Extrait 3 (épisode 1.2.)

Au début de la deuxième phase de la séance, l'écriture du compte rendu passe par un épisode de discussion de l'orthographe des mots qu'ils sont en train d'écrire (extrait 4). Nous considérons qu'il s'agit là d'un aspect « technique » de l'écriture par rapport à la tâche prévue; ce ne serait évidemment pas une interprétation possible dans le cas d'une séance d'apprentissage de la langue. Cet épisode est donc assez comparable - malgré des modalités d'activité très différentes - à celui du déballage des glaçons avec un glissement sur les aspects techniques de la tâche.

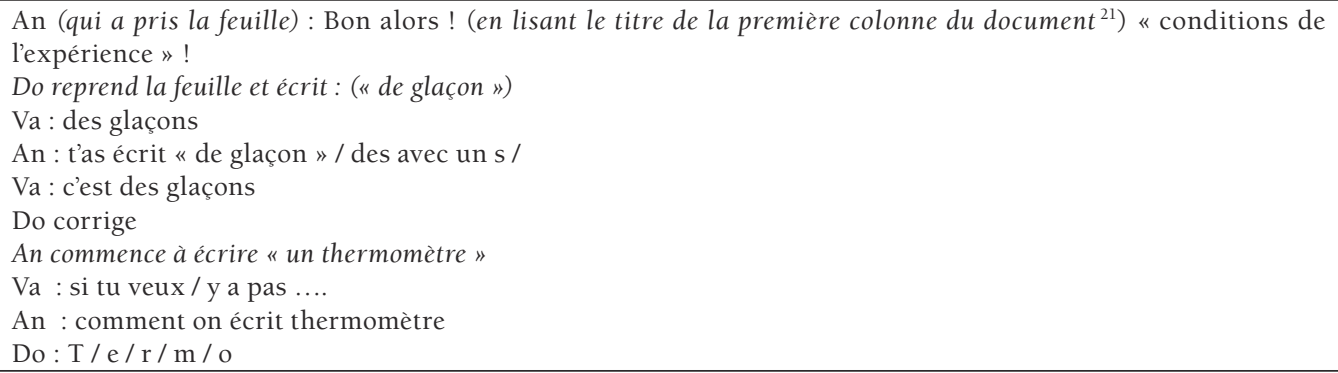

Extrait 4 (épisode 2.1.)

Une intervention de l'enseignant (extrait 5) va une nouvelle fois recentrer l'activité vers une dimension plus "scientifique ", en mettant provisoirement de côté les problèmes de langue ("ne faites pas de phrases"). Elle va aussi essayer de focaliser l'attention des élèves sur les aspects essentiels du compte-rendu (ce qu'on a fait et surtout ce qu'on a observé), ce qui pose quelques problèmes aux élèves dans un premier temps. L'accompagnement des élèves dans ce délicat travail d'écriture permet de revenir assez rapidement sur la tâche attendue. 


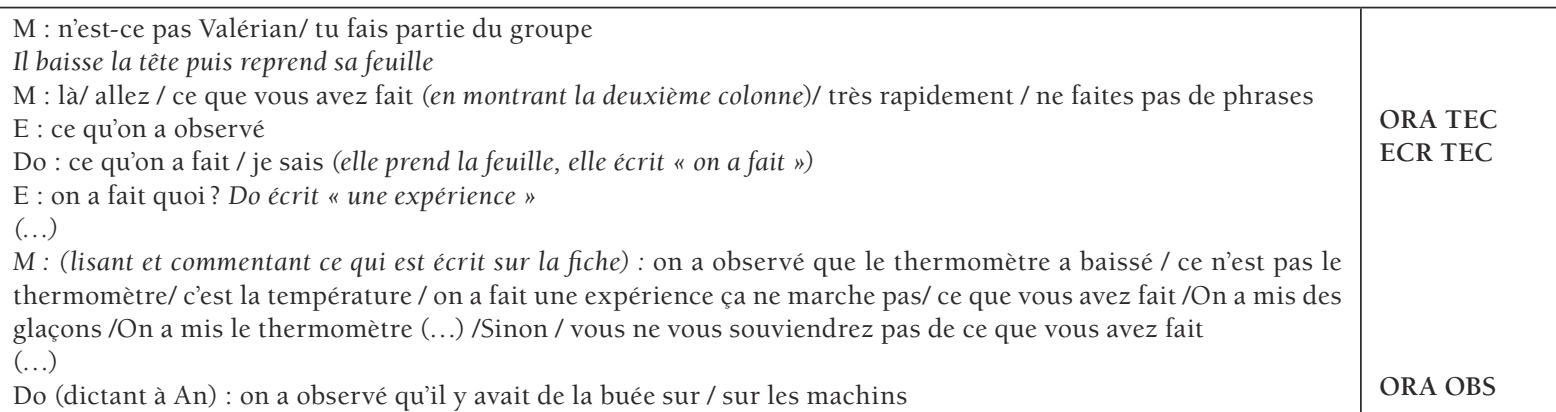

Extrait 5 (passage de l'épisode 2.2. à l'épisode 2.3.)

Ces quelques extraits nous semblent assez représentatifs du décalage entre ce qui est visé par l'enseignant et ce que font effectivement les élèves. Ils montrent aussi l'importance des interventions du maître pour recentrer l'activité vers les tâches prévues. Nous discuterons plus loin des différents types d'intervention du maître. Nous allons nous intéresser pour l'instant à l'activité effective des élèves que nous avons pu observer dans les différentes phases du travail. Nous présentons dans le tableau reproduit en Annexe le récapitulatif de l'analyse réalisée sur l'ensemble du corpus. Plusieurs résultats peuvent être tirés de cette analyse :

- le type théorique est peu observé dans ces deux phases de travail ( 5 occurrences) et uniquement à l'oral en présence du maître,

- l'activité effective des élèves se situe majoritairement dans les types technique (31 occurrences) et observationnel (29 occurrences),

- la répartition des modalités d'action est globalement conforme aux tâches prescrites avec un grand nombre d'actions matérielles dans la première phase et un grand nombre d'actions d'écriture dans la seconde phase, les actions orales ${ }^{22}$ étant nombreuses dans l'ensemble du corpus analysé,

- les «sorties» de l'activité prévue, assez nombreuses (17), sont observées principalement pendant l'épisode de réalisation effective de l'expérimentation et plus ponctuellement surtout en début ou fin d'épisode,

- la répartition entre les deux types principaux d'activité est très variable selon les épisodes : les épisodes de début de chaque phase sont presque exclusivement dans le type technique (épisodes 1.1. et 2.1.), alors que les épisodes suivants montrent une part de type observation beaucoup plus importante,

- ce sont les interventions du maître qui produisent ce changement d'activité effective : cela apparaît dans le tableau par un déplacement significatif des types d'activité vers la gauche après chaque intervention du maître.

Avant de revenir sur ce dernier résultat qui est certainement le plus marquant, nous souhaitons ajouter quelques remarques. L'absence d'activité de type théorique pendant les épisodes de travail autonome indique que les élèves ne reviennent pas spontanément sur l'enjeu cognitif des tâches prescrites - enjeu qui était de tester des modèles explicatifs (cf. document 2). Les élèves respectent en fait a minima les tâches prescrites en se limitant aux aspects observationnels et surtout techniques dans un premier temps. Par ailleurs, il faut relativiser l'importance des sorties de tâches : en effet, ces « sorties » ont souvent été très brèves et n'ont concerné en général qu'un ou deux élèves ${ }^{23}$. Elles n'ont donc pas empêché le déroulement d'une activité globalement conforme aux prescriptions ${ }^{24}$.

\section{Rôle des interventions du maître}

Comme nous venons de l'indiquer, les types d'activité effective des élèves sont sensiblement déplacés par les interventions du maître. L'analyse du rôle de ces interventions dans la régulation de l'activité des élèves et dans les interactions entre pairs a été présentée plus en détail par ailleurs 


\section{ANALYSE DidACTIQUe de L'ACTIVIté EFFECTIVE DES ÉLÈVES EN SCIENCES À L'ÉCOLE PRIMAIRE}

\section{Joël Bisault E Christine Berzin}

(Berzin \& Bisault, 2007) ; nous ne retiendrons ici que les résultats principaux de cette analyse. Cette analyse a donné lieu à un codage des interventions verbales selon une grille qui emprunte un certain nombre de catégories à l'analyse des interactions de tutelle de Bruner (1983). Les différentes catégories envisagées et les codages utilisés dans notre analyse sont présentés dans le document ci-dessous.

\begin{tabular}{|l|l|}
\hline $\begin{array}{l}\text { Interventions visant à définir la } \\
\text { tâche à réaliser }\end{array}$ & En terme d'objectif final à atteindre : Obj \\
\cline { 2 - 2 } & En terme d'organisation matérielle de l'expérimentation à mettre en œuvre : Org \\
\hline $\begin{array}{l}\text { Interventions visant à expliciter } \\
\text { ou reformuler un énoncé } \\
\text { précédemment formulé }\end{array}$ & Explicitation de la consigne visant à définir l'objectif à atteindre : Expl \\
\cline { 2 - 2 } & Reformulation de propos préalablement énoncés par les élèves ou par la maîtresse : Ref \\
\hline $\begin{array}{l}\text { Interventions portant sur le } \\
\text { cours de la réalisation de la } \\
\text { tâche }\end{array}$ & Signalisation de caractéristiques déterminantes pour la réalisation de la tâche : Sign \\
\cline { 2 - 2 } & Maintien de l'orientation : Mor \\
\cline { 2 - 2 } & Remise en cause (objectif - point ponctuel - vocabulaire) : Rem \\
\hline $\begin{array}{l}\text { Régulation / conditions } \\
\text { d'exercice de la tâche }\end{array}$ & Rappel à l'ordre : Rap \\
\hline
\end{tabular}

Document 6 : Grille d'analyse des interventions du maître

Les résultats de cette analyse sont reportés dans le tableau ci-dessous qui reprend aussi les observations des différents types d'activité effective des élèves. Contrairement au document présenté dans l'Annexe, les activités effectives des élèves n'ont pas été distinguées selon leur modalité de réalisation (orale, écrite, matérielle) et ont donc été regroupées pour chaque épisode. Le tableau du document 7 présente le nombre d'occurrences des catégories de chacune des grilles d'analyses pour chacun des épisodes définis précédemment.

\begin{tabular}{|c|c|c|c|c|c|c|c|c|c|c|c|c|}
\hline & \multicolumn{8}{|c|}{ Interventions du maître } & \multicolumn{4}{|c|}{$\begin{array}{c}\text { Types d'activité effective } \\
\text { des élèves }\end{array}$} \\
\hline \multicolumn{13}{|l|}{ Phase 1 : expérimentation } \\
\hline 1.1. « déballage des glaçons » & & $\begin{array}{c}\text { Org } \\
1\end{array}$ & & & & & & & & & $\begin{array}{c}\text { Tec } \\
9\end{array}$ & $\begin{array}{c}\text { Sor } \\
2\end{array}$ \\
\hline 1.2. intervention du maître & $\begin{array}{c}\text { Obj } \\
1\end{array}$ & $\begin{array}{c}\text { Org } \\
3\end{array}$ & & $\begin{array}{c}\text { Ref } \\
3\end{array}$ & $\begin{array}{c}\text { Sign } \\
1 \\
\end{array}$ & $\begin{array}{c}\text { Mor } \\
2\end{array}$ & & & $\begin{array}{c}\text { The } \\
1\end{array}$ & $\begin{array}{c}\text { Obs } \\
1\end{array}$ & $\begin{array}{c}\text { Tec } \\
2 \\
\end{array}$ & \\
\hline 1.3. expérimentation effective & & & & & & $\begin{array}{c}\text { Mor } \\
1\end{array}$ & & $\begin{array}{c}\text { Rap } \\
5\end{array}$ & & $\begin{array}{c}\text { Obs } \\
9\end{array}$ & $\begin{array}{c}\text { Tec } \\
7\end{array}$ & $\begin{array}{c}\text { Sor } \\
11\end{array}$ \\
\hline 1.4. intervention du maître & $\begin{array}{c}\text { Obj } \\
3\end{array}$ & & $\begin{array}{c}\text { Expl } \\
1\end{array}$ & $\begin{array}{c}\operatorname{Ref} \\
5\end{array}$ & $\begin{array}{c}\text { Sign } \\
3\end{array}$ & $\begin{array}{c}\text { Mor } \\
2\end{array}$ & & $\begin{array}{c}\text { Rap } \\
1\end{array}$ & $\begin{array}{c}\text { The } \\
3\end{array}$ & $\begin{array}{c}\text { Obs } \\
2\end{array}$ & & \\
\hline \multicolumn{13}{|l|}{ Phase 2 : écriture du compte rendu } \\
\hline 2.1. «remplissage de la fiche» & & & $\begin{array}{c}\text { Expl } \\
2\end{array}$ & $\begin{array}{c}\text { Ref } \\
3\end{array}$ & $\begin{array}{c}\text { Sign } \\
1\end{array}$ & & & & & $\begin{array}{c}\text { Obs } \\
2\end{array}$ & $\begin{array}{c}\text { Tec } \\
7\end{array}$ & $\begin{array}{c}\text { Sor } \\
2\end{array}$ \\
\hline 2.2. intervention du maître & $\begin{array}{l}\text { Obj } \\
2\end{array}$ & & $\begin{array}{c}\text { Expl } \\
2\end{array}$ & $\begin{array}{c}\text { Ref } \\
1\end{array}$ & & & & $\begin{array}{c}\text { Rap } \\
1\end{array}$ & & & $\begin{array}{c}\text { Tec } \\
1\end{array}$ & \\
\hline 2.3. rédaction effective de la fiche & & & & & & & & & & $\begin{array}{c}\text { Obs } \\
4\end{array}$ & $\begin{array}{c}\text { Tec } \\
2\end{array}$ & \\
\hline 2.4. intervention du maître & $\begin{array}{c}\text { Obj } \\
3\end{array}$ & & & $\begin{array}{c}\text { Ref } \\
2\end{array}$ & & & $\begin{array}{c}\text { Rem } \\
4\end{array}$ & & $\begin{array}{c}\text { The } \\
1\end{array}$ & $\begin{array}{c}\text { Obs } \\
6\end{array}$ & $\begin{array}{c}\text { Tec } \\
2\end{array}$ & \\
\hline 2.5. fin de la rédaction de la fiche & & & & & & & & $\begin{array}{c}\text { Rap } \\
1\end{array}$ & & $\begin{array}{c}\text { Obs } \\
5\end{array}$ & $\begin{array}{c}\text { Tec } \\
1\end{array}$ & $\begin{array}{c}\text { Sor } \\
2\end{array}$ \\
\hline Total général & $\begin{array}{c}\text { Obj } \\
9 \\
17 \%\end{array}$ & $\begin{array}{c}\text { Org } \\
4 \\
7 \%\end{array}$ & $\begin{array}{c}\text { Expl } \\
5 \\
10 \%\end{array}$ & $\begin{array}{c}\text { Ref } \\
14 \\
25 \%\end{array}$ & $\begin{array}{c}\text { Sign } \\
5 \\
10 \%\end{array}$ & $\begin{array}{c}\text { Mor } \\
5 \\
10 \%\end{array}$ & $\begin{array}{c}\text { Rem } \\
4 \\
7 \%\end{array}$ & $\begin{array}{c}\text { Rap } \\
8 \\
14 \%\end{array}$ & $\begin{array}{c}\text { The } \\
5 \\
6 \%\end{array}$ & $\begin{array}{c}\text { Obs } \\
29 \\
35 \%\end{array}$ & $\begin{array}{c}\mathrm{Tec} \\
31 \\
38 \%\end{array}$ & $\begin{array}{c}\text { Sor } \\
17 \\
21 \%\end{array}$ \\
\hline
\end{tabular}

Document 7 : Récapitulatif des interventions du maître ${ }^{25}$ et des différents types d'activité effective des élèves 
Le tableau donne un aperçu global de la nature des interventions du maître. Nous observons une prédominance des reformulations de propos préalablement énoncés à part égale par les élèves ou par la maîtresse ( $25 \%$ de l'ensemble) et des interventions visant à définir la tâche en terme d'objectif à atteindre (17\%) - interventions qui interviennent principalement à chaque changement de tâche -. Ces catégories prédominantes sont suivies par les explicitations complémentaires de ces objectifs (10\%), les indications visant à maintenir la poursuite de ces objectifs (10\%) ou à l'attention des élèves sur les caractéristiques déterminantes pour la réalisation de la tâche (10\%) qui interviennent cette fois plutôt en cours de réalisation de tâche. On notera également la présence d'un certain nombre de rappels à l'ordre (14\%), les deux catégories restantes ne représentant quant à elles pas plus de $7 \%$ de l'ensemble des interventions. La mise en relation des analyses des interventions du maître et de l'activité des élèves montre que ces interventions ont notamment permis aux élèves de revenir à deux reprises sur l'objectif initial de la tâche et de changer de type d'activité comme le montrent par exemple les activités développées à l'issue des interventions du maître faisant suite à l'épisode « déballage des glaçons » de la première phase ou à l'épisode « remplissage de la fiche " de la deuxième phase. On peut observer dans les deux cas un déplacement vers la gauche dans les quatre dernières colonnes du tableau.

Dans le premier exemple (passage du premier épisode au troisième épisode de la phase d'expérimentation) la mise en ouvre de l'expérience se caractérise par de nombreuses manipulations pouvant s'accompagner le cas échéant de conflits, voire de sorties de tâches. Cette centration sur le faire (l'activité technique) plutôt que sur le comment faire (la prise de conscience des propriétés de l'activité) conduit les élèves à perdre de vue l'objectif fixé. Le rappel de cet objectif par le maître s'avère un élément indispensable à la reprise effective de l'expérience. Alors que l'épisode précédant n'avait laissé place qu'à une activité technique (TEC), on assiste alors à une activité portant majoritairement sur les observations réalisées (OBS).

De la même manière qu'au cours de la première phase, l'intervention du maître après l'épisode 1 de la seconde phase (écriture du compte-rendu) apparaît déterminante en permettant comme précédemment un déplacement du type d'activité des élèves. En se recentrant sur l'objectif poursuivi, ces derniers parviennent à se départir de préoccupations centrées sur l'orthographe et la répartition des rôles au niveau de la tâche concrète d'écriture plutôt que sur la teneur des observations réalisées. Ils réussissent ainsi à revenir à la tâche attendue et à formaliser par écrit leurs observations (épisode 2.3.).

L'influence des interventions du maître sur l'activité effective des élèves n'a été qu'esquissée dans cet article. On peut noter que l'essentiel des interventions du maître vise un guidage de l'activité des élèves que cette activité soit matérielle ou langagière. Pour autant le maître ne fait jamais à la place de l'élève ni ne dit directement ce que l'élève devrait trouver. On retrouve là le phénomène de "réticence didactique » caractéristique d'une certaine forme d'interaction langagière entre le professeur et les élèves (Sensevy \& Quillio, 2002). Le rôle des interventions du maître dans les apprentissages de l'élève a fait l'objet de nombreuses études didactiques qui ont montré l'importance des interactions didactiques entre les professeurs et les élèves et les différentes formes que pouvait prendre cette interaction (Dumas Carré \& Weil Barais, 1998). Plus récemment, des travaux ont été réalisés dans le domaine des mathématiques au collège (Chappet Parries, 2004) mais la comparaison avec nos travaux est assez difficile car les tâches « disciplinaires » et les conditions de fonctionnement didactiques sont très différentes. Le fonctionnement observé ici nous semble relever d'une approche curriculaire de type " processdriven " alors que les fonctionnements observés dans le secondaire nous semblent relever d'une approche de type "content-driven " si on reprend la distinction proposée par Ross (2000). De la même façon, le rapprochement avec des travaux récents menés en didactique de la physique au lycée (Tiberghien et al., 2007) est discutable que ce soit sur le plan des objets étudiés (rythme d'introduction des savoirs) ou sur celui de la visée générale de la recherche (comparaison de pratiques de classe) ; en revanche, on peut remarquer dans cette dernière recherche une certaine similitude des données utilisées (actions du professeur et des élèves) et des échelles d'observation (mésoscopique et microscopique). La spécificité des modes de fonctionnement de l'école primaire nous incite à préférer des comparaisons avec des domaines disciplinaires a priori éloignés mais pour le même niveau 
scolaire; c'est une piste que nous explorons actuellement dans le prolongement d'autres travaux à visée comparatiste (Bisault \& Le Bourgeois, 2006).

\section{Prégnance des aspects techniques de la tâche}

Dans cette séance, la tâche prescrite pour la première phase de travail a été formulée par le maître lors de la présentation collective des affiches ( «... on va essayer de faire du brouillard, on va essayer de reproduire dans la classe les conditions qui permettent de faire du brouillard à l'extérieur... »). Comme nous l'avons déjà indiqué, cette tâche doit a priori conduire à la mise en relation du registre empirique (technique et observationnel) et du registre des modèles (registre théorique). En premier lieu, l'expérience s'inscrit dans une recherche d'explication, comme l'ensemble des activités menées dans cette séquence qui doivent permettre de répondre à deux questions : questions Q1 « qu'est-ce que le brouillard?» et Q2 « comment se forme-t-il? ». On peut ainsi considérer que les élèves vont tester expérimentalement les modèles explicatifs provisoires qu'ils ont ébauchés dans la séance précédente et qui sont résumés dans la première partie de leurs affiches (cf. document 1). Cependant, cette séance est centrée sur la réalisation matérielle de l'expérience et sur la conduite d'observations, comme l'indique très clairement la fiche devant être remplie par les élèves dans la deuxième phase de la séance fiche qui comporte trois colonnes: "les conditions de l'expérience, ce qu'on a fait, ce qu'on a observé ». Cette séparation, assez classique pour un compte-rendu scolaire d'expérience, doit conduire à distinguer trois types de renseignements: les éléments matériels nécessaires à la réalisation de l'expérience, les actions qu'ils ont effectuées avec ce matériel et les observations qu'ils ont pu faire. La tâche prescrite dans cette séance est donc très complexe puisqu'il existe deux types de mises en tension. En premier lieu, au niveau de l'ensemble de la séquence didactique, l'expérience met en tension une tâche théorique et une tâche empirique correspondant à deux registres différents de la modélisation du brouillard. En second lieu, à l'échelle de la séance observée, la tâche empirique est elle-même dédoublée entre une tâche d'observation et une tâche technique. Enfin, la tâche se décline selon trois modalités complémentaires (action matérielle, discussion orale, écriture) dont l'importance relative s'inverse entre la première et la seconde phase de la séance. Dans ces conditions la tâche prescrite est très complexe et peut ainsi entraîner des interprétations multiples au niveau de l'activité effective des élèves.

Il est assez clair, que les aspects techniques dominent dans les activités effectives des élèves (31 occurrences pour le type technique) mais que les activités de type observation sont également nombreuses (29 occurrences) principalement à la suite des interventions du maître. En revanche, les activités dans le type théorique sont peu nombreuses ( 5 occurrences) et se produisent toujours en présence du maître, ce qui montre clairement que le maître doit rappeler l'enjeu explicatif de cette expérience, enjeu que les élèves ont semble-t-il tendance à perdre de vue à certains moments. Par ailleurs, l'importance relative des activités dans le type technique montre que les élèves peuvent aussi perdre de vue à certains moments l'enjeu observationnel de l'expérience en se concentrant sur les aspects manipulatoires. C'est sans doute un détour inévitable, y compris dans le travail des chercheurs, mais à certains moments, les élèves semblent se faire piéger par certains aspects de la tâche qui peuvent rentrer en interaction avec d'autres moments familiers dans le domaine scolaire ou en dehors de l'école. Ainsi, le « déballage » des glaçons à partir des sacs à glaçons (sacs jetables à remplir d'eau) est indispensable dans un usage domestique ordinaire mais il n'a pas d'intérêt si on utilise les glaçons comme "source froide ». Les élèves ont donc perdu beaucoup de temps avant l'intervention de la maîtresse : "vous avez oublié votre expérience pour vous amuser avec les glaçons» (extrait 2). De la même façon, l'épisode de remplissage de la fiche comporte plusieurs échanges entre les élèves sur la question d'écrire ou non son nom de famille. Un peu plus loin une discussion est engagée sur l'orthographe du mot thermomètre (extrait 4), discussion arrêtée par la maîtresse qui signale que l'orthographe n'est pas importante pour le moment. Il est clair que ces deux aspects de l'écriture (nom de l'élève, orthographe) sont très importants pour d'autres moments scolaires : c'est donc le contrat scolaire « ordinaire » qui piège ici les élèves. L'activité de type technique apparaît donc comme un régime de fonctionnement habituel pour les élèves - que cette habitude relève du quotidien scolaire ou du quotidien non scolaire. En agissant ainsi, les élèves respectent donc, d'une certaine manière au moins, la tâche prescrite. Ce 
n'est pas le cas pour certaines activités qui résultent manifestement d'une sortie de la tâche quand par exemple Valérian donne un message à Annie sur un Post-it ou quand Annie joue avec une peluche. Les élèves ne sont pas tous également concernés par ces comportements «non scolaires » qui sont très bien identifiés et pris en compte par le maître (« n'est-ce pas Valérian/ tu fais partie du groupe » - extrait 5).

Si on considère l'influence des interventions du maître sur le type d'activité effective des élèves (cf. tableau de résultats en annexe) on peut observer quatre régimes différents :

- un « régime spontané » (en l'absence du maître) dans le type technique, lié à la prise en compte de certains aspects techniques de la tâche - régime qu'on retrouve à chaque changement de matériel (épisode 1.1. «Déballage des glaçons » ou épisode 2.1. « Remplissage de la fiche »),

- un "régime régulé », dans le type observation, obtenu après intervention de recentrage du maître (épisode 1.3. «Expérimentation effective » ou épisode 2.3. "Rédaction effective de la fiche »,

- un « régime forcé », dans le type explicatif, presque uniquement sous la présence « en continu » du maître (épisode 1.2, 1.4 et 2.4)

- un « régime de rupture » quand certains élèves sortent complètement de la tâche. Il est à noter que ce régime se produit logiquement quand le maître est absent.

\section{Interactions entre les moments scientifiques scolaires et d'autres moments}

L'analyse de l'activité effective des élèves dans ce travail expérimental fait apparaître ce qu'on peut appeler un glissement de l'activité par rapport à ce qui est visé par l'enseignant. Ce glissement, présent notamment lors de la réalisation matérielle de l'expérience, semble provenir essentiellement de l'ambiguité de la tâche impliquant un dispositif matériel. En se polarisant sur la réalisation matérielle de l'expérience l'élève oublie l'enjeu cognitif de l'activité : l'activité matérielle n'est plus un moyen mais une fin en soi. On peut considérer que les élèves sortent ainsi (involontairement) de l'activité scientifique scolaire pour rentrer dans une activité « ordinaire» (activité domestique par exemple) où la manipulation d'objets répond à une finalité utilitaire. Ce glissement que nous avons observé est très voisin de ceux observés dans d'autres domaines d'apprentissage et qui sont considérés comme des effets du contrat didactique (Bautier, 2006).

Cette difficulté observée ici dans un cas particulier nous semble liée à une des caractéristiques de ce domaine d'enseignement de l'école primaire (et plus généralement de ce qui relève de la « découverte du monde ») qui est de porter sur des objets d'étude faisant partie de l'environnement familier des élèves. La familiarité des élèves avec certains objets ou phénomènes peut constituer une difficulté quand l'utilisation qui est faite de ces objets dans les moments scolaires de sciences s'oppose à leur utilisation habituelle (Bisault, 2005b). C'est donc bien la distinction entre des « moments scientifiques scolaires " amenant à porter un regard particulier sur certains objets et d'autres moments portant sur les mêmes objets qui peut poser des difficultés aux élèves. La familiarité avec ces objets peut faciliter la mise en place d'activités scientifiques sur le plan pratique mais elle peut aussi être un piège redoutable pour les élèves comme pour les enseignants.

Un glissement comparable à celui que nous venons de décrire se produit dans la phase d'écriture du compte-rendu mais cette fois-ci l'ambiguitté de la tâche ne correspond pas à un lien possible entre moment scientifique scolaire et moment non scolaire mais plutôt à un lien fait par les élèves entre deux moments scolaires différents comprenant une tâche d'écriture : ceux insérés dans une séquence d'apprentissage scientifique et ceux relevant d'un apprentissage de la langue. Ce n'est qu'après une intervention du maître que les élèves abandonnent le travail " technique " d'écriture (orthographe et forme des phrases notamment) pour porter le travail d'écriture sur les phénomènes observés (cf. extrait 5). Le travail d'écriture attendu ici exige donc une certaine forme de renoncement par rapport à des aspects importants $\mathrm{du}$ « contrat scolaire », du moins tel qu'il peut être perçu par les élèves, notamment dans le respect de certaines «normes » d'écriture. Cette difficulté a déjà été mise en évidence dans des recherches antérieures sur l'écriture en sciences qui ont montré la nécessité 
de revaloriser le rôle des brouillons par rapport aux écrits expositifs répondant à des exigences formelles (Vérin, 1995).

C'est sans doute à cause d'une prise en compte insuffisante des spécificités "scientifiques » des tâches de manipulation et d'écriture que les élèves ont accordé autant d'importance aux aspects «techniques ». L'entrée dans ce moment scolaire de sciences exige donc un double renoncement. D'une part, il faut renoncer à agir sur des objets pour obtenir des effets immédiats comme c'est le cas dans une activité quotidienne mais plutôt agir sur des objets pour observer et expliquer des phénomènes. D'autre part, les élèves doivent renoncer à « réussir » la production formelle de textes comme c'est le cas dans d'autres moments scolaires mais écrire pour construire des explications. Pour que l'activité scientifique scolaire joue pleinement son rôle, il est donc nécessaire de la distinguer à la fois des activités non scolaires et d'autres activités scolaires.

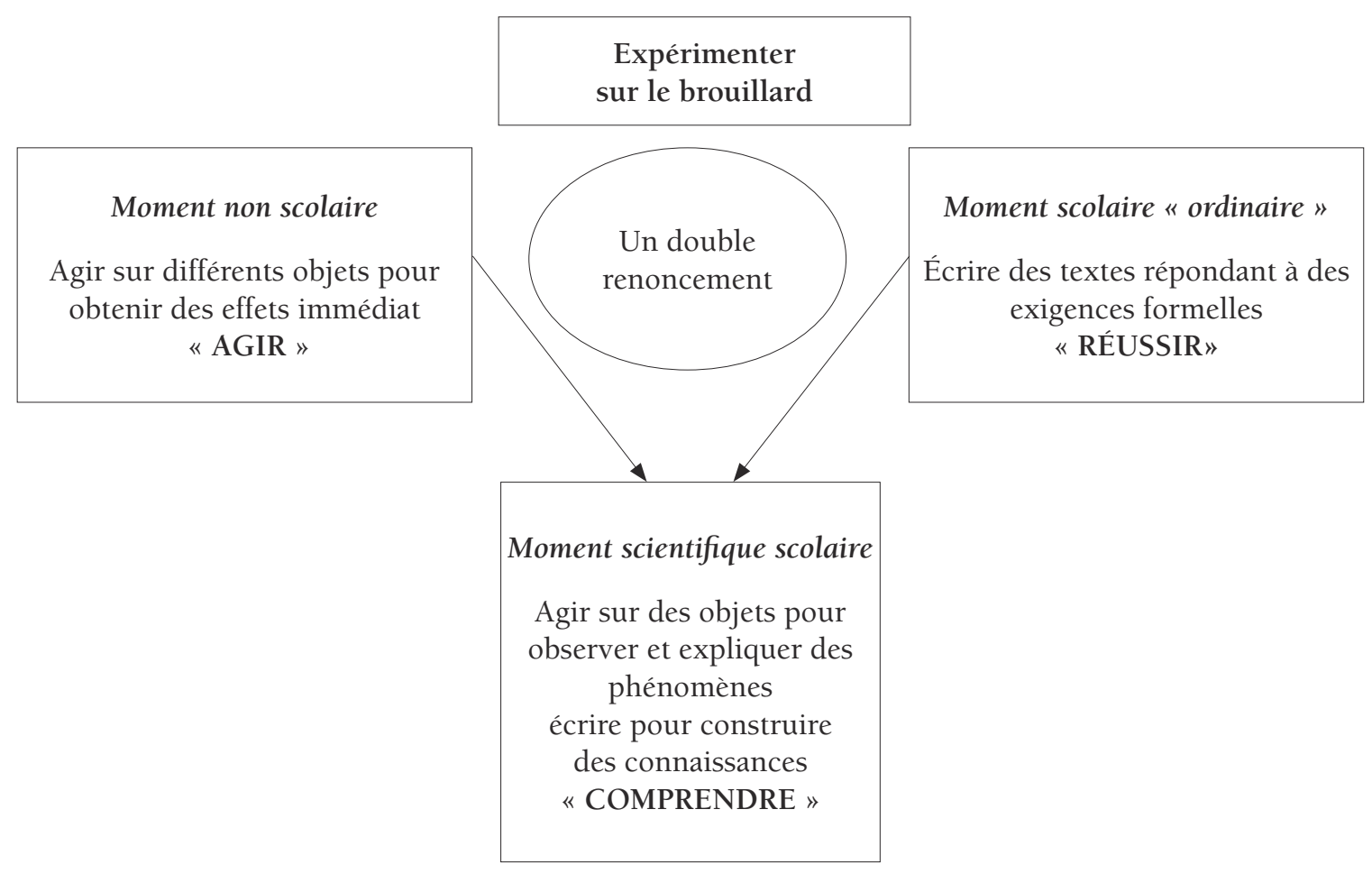

Document 8 : moment non scolaire, moment scolaire ordinaire ou moment scientifique scolaire

\section{Conclusion}

Cette recherche reste exploratoire et les données recueillies sont trop limitées pour permettre une généralisation ou constituer une quelconque validation. Cependant, nos observations soulèvent un certain nombre de questions éducatives qui ne sont pas nouvelles mais qui restent encore en partie au moins inexplorées. En effet, une partie des difficultés rencontrées par les élèves dans nos observations est liée à l'interprétation des tâches prescrites. La complexité des tâches avec l'existence de différentes modalités d'activité et de différents registres complique ce travail d'interprétation. Cette difficulté d'interprétation d'une tâche complexe observée ici dans un cas particulier d'enseignement scientifique à l'école primaire se retrouve dans d'autres domaines d'enseignement et pour d'autres niveaux scolaires. Dans un tour d'horizon des pratiques scolaires actuelles, Bautier (2006) montre que 
les élèves peuvent être induits en erreur par l'utilisation scolaire d'objets familiers à cause de la pluralité des registres sur lesquels ces objets peuvent être saisis. Ainsi, certains élèves privilégient l'accomplissement formel de la tâche en restant sur un registre de travail immédiat sur les objets scolaires manipulés. Cette notion de travail immédiat nous paraît intéressante pour caractériser les tâches que nous étudions ici : en effet, la manipulation d'objets matériels pour réaliser « techniquement » une expérience ou l'inscription d'un compte-rendu sur une feuille doivent à la fois produire un résultat immédiat mais aussi être mises en relation avec la dimension cognitive de l'activité. C'est à cette condition que les activités proposées peuvent réellement contribuer à des apprentissages : comme le mentionne Bautier (2006), une « secondarisation » des objets scolaires est nécessaire pour dépasser le travail immédiat réduit à sa transparence. L'identification précise des «systèmes de tâches » mis en ouvre par les enseignants à différents grains de temporalité est donc une piste de recherche importante (Sensevy, 2007).
Le croisement d'enjeux disciplinaires et d'enjeux d'apprentissages transversaux (maîtrise de la langue ou éducation civique) est une source de difficulté pour les élèves. Dans le cas observé ici, l'activité d'écriture peut être interprétée par les élèves comme un moment de travail scolaire sur la langue ou comme un moyen au service de l'activité scientifique. Les échanges oraux entre élèves peuvent aussi être l'objet d'interprétations multiples ${ }^{26}$. Nous rejoignons ici l'analyse de Sensevy et Turco $(2004$, p. 2) qui considèrent que la «polyfonctionnalité » des moments de débat est source de multiples ambiguités; le débat est en effet à la fois un moment essentiel dans l'élaboration des savoirs scientifiques, un moment de mise en œuvre de compétences langagières relevant notamment de l'argumentation mais aussi un lieu privilégié pour l'éducation civique des élèves. Pour bien comprendre la réalité des moments scolaires de "sciences et technologie ", il paraît donc nécessaire d'analyser les continuités, ruptures ou complémentarités entre ces moments et d'autres moments scolaires ou non scolaires qui peuvent interagir avec eux. C'est une piste de réflexion que nous avons commencé à explorer dans une nouvelle recherche ${ }^{27}$. 


\section{NOTES}

1. BO hors-série du 12 avril 2007 (Mise en œuvre du socle commun de connaissances et de compétences - horaires et programmes d'enseignement de l'École Primaire).

2. Classe de CM1 : élèves de 9-10 ans. La séquence didactique a été conçue et menée par Madame Danièle Badia, professeur des écoles - maître formateur, en collaboration avec Monsieur Vincent Fontaine professeur de sciences physiques à l'IUFM de l'Académie d'Amiens.

3. Dans une étude précédente portant sur la même séquence de classe (Fontaine, Vérin \& Bisault, 2005), nous avons au contraire étudié l'évolution des connaissances des élèves tout au long de la séquence en utilisant d'autres types de données et un autre grain d'analyse.

4. Nous prenons ici le terme objet dans une acception large; nous reviendrons à la fin de cet article sur certaines caractéristiques de ces « objets ».

5. Ces phases de travail constituent des « moments scolaires » bien identifiables par les élèves (Lebeaume, 2000) aussi bien dans leurs aspects spécifiques au domaine d'apprentissage considéré (paramètres intrinsèques) que dans leurs aspects plus organisationnels (paramètres extrinsèques).

6. Un mode d'alternance voisin est couramment utilisé à l'école primaire dans d'autres champs disciplinaires tels que l'histoire par exemple (Bisault \& Le Bourgeois, 2006). En revanche, ce mode d'alternance est très différent de l'alternance entre séances de cours et séances de travaux pratiques généralement pratiquée dans l'enseignement scientifique au niveau secondaire, en physique par exemple (Tiberghien, Malkoun, Buty, Souassy, \& Mortimer, 2007).

7. On doit distinguer trois échelles : - celle de l'observation courante non « instrumentée » (échelle macroscopique) - celle des gouttelettes d'eau trop petites pour être visibles à l'œil nu mais qui ont un effet visible à l'échelle macroscopique (échelle mésoscopique) - celle des atomes et des molécules (échelle microscopique).

8. Le qualificatif « réel » est utilisé en didactique du curriculum en opposition au curriculum formel ou potentiel par certains auteurs (Martinand, 2003) alors que d'autres auteurs utilisent l'opposition entre curriculum prescrit et curriculum effectif (Audigier, Crahay, \& Dolz, 2006). Cependant, ces désignations concernent généralement des analyses à une échelle beaucoup plus large que celle que nous proposons dans cet article Notre échelle d'analyse est en revanche comparable à celle de certains travaux s'inscrivant dans le cadre d'une approche ergonomique qui tiennent comme essentielle la distinction entre tâche prescrite et activité effectivement réalisée (Leplat, 1997).

9. Il faut prendre ici le terme "texte » dans le sens de toute production langagière qu'elle soit écrite ou orale.

10. Nous n'aborderons pas ici la distinction entre activité et pratique, théorisée également par plusieurs de ces auteurs - distinction que nous avons prise en compte dans une autre recherche (Bisault, 2005c).

11. C'est en revanche une piste d'analyse que nous avions explorée pour un autre domaine d'apprentissage et un autre niveau scolaire : l'étude du fonctionnement d'un objet technique à l'école maternelle (Bisault, 2005b). Nous avions alors intégré les gestes symboliques dans l'ensemble des manifestations sémiotiques analysées ; ces gestes peuvent constituer dans certains cas un langage très efficace notamment quand les phénomènes étudiés ont une forte dimension spatiale et quand les élèves maîtrisent mal les codes verbaux.

12. Il est en effet important de noter que dans certaines phases de cette séquence didactique, la responsabilité du savoir est largement laissée aux élèves, situation fort différente de ce qu'on peut observer par exemple pendant un cours de physique dans le secondaire (Tiberghien et al., 2007).

13. Il faut comprendre ce terme dans le sens d'action matérielle observable par le chercheur au travers d'un ensemble de gestes des élèves et/ou de modifications de leur environnement.

14. Notons que la combinaison MAT THE ne semble pas réalisable a priori.

15. Les prénoms des élèves ont été changés pour cet article. 
16. Le contenu de l'affiche a été retranscrit pour une meilleure lisibilité mais l'orthographe et la présentation ont été conservées.

17. La première séance de travail a alterné différentes phases de travail (individuel, collectif et en groupes) qui ont permis l'expression des idées préalables des élèves et une première discussion guidée par le maître sur la nature et les causes du phénomène étudié. Cet écrit intermédiaire n'est donc ni tout à fait un écrit « premier » ni un écrit terminal validé par le maître ; c'est au contraire un écrit de travail qui s'inscrit de façon fonctionnelle dans la démarche d'investigation, le processus d'écriture étant associé au processus d'élaboration collective des connaissances (Vérin, 1995). Les discussions qui ont accompagné l'écriture de ces protocoles ont été analysées par ailleurs pour deux autres groupes de la même classe (Bisault, 2008).

18. Il n'a pas été tenu compte des interventions auprès des autres groupes car elles étaient généralement peu audibles dans l'enregistrement (donc vraisemblablement peu audibles par le groupe observé). On ne peut évidemment pas exclure une certaine influence de ces interventions "périphériques».

19. Nous avons choisi de considérer ces deux moments comme constituant un seul épisode, d'une part parce que le deuxième moment était très court (quelques secondes) et d'autre part parce qu'il y a eu superposition de ces deux moments, la présentation de la fiche par l'enseignant étant faite pendant la discussion des résultats.

20. Conventions de retranscription utilisées:

E : élève non identifié - EE : plusieurs élèves en même temps - M : maître - XX : inaudible - (..) : coupure dans la retranscription - en italiques : formulation des actions matérielles observées.

21. Le compte-rendu d'expérience doit être rédigé sur un document sur lequel figurent déjà trois colonnes: "les conditions de l'expériencel ce qu'on a fait/ ce qu'on a observé ".

22. Il faut rappeler qu'au travers de nombreuses fonctions différentes, l'oral joue un rôle extrêmement important dans la classe (Nonnon, 2001).

23. Cela pose la question du rapport à l'activité et sans doute du rapport au savoir de chacun des élèves (Venturini, 2007). Nos observations ne permettent pas d'avancer de résultat précis sur ce problème car comme nous l'avons déjà indiqué - notre analyse a porté sur l'activité collective, les actions individuelles n'étant considérées que comme des indicateurs de cette activité collective.

24. Nous ne discuterons pas dans cet article du fonctionnement de la classe sur le plan pédagogique, mais la comparaison avec d'autres classes nous amène à penser qu'il s'agit d'un fonctionnement satisfaisant avec une bonne maîtrise pédagogique du maître. Nous avons noté par exemple que les interventions du maître suivaient généralement de très près les « sorties »/débordements - ce qui indique une bonne observation du comportement des élèves ou une bonne anticipation.

25. Les moments de travail dirigés par le maître sont indiqués en grisé dans le tableau. Des interventions plus ponctuelles du maître sont également observées dans les épisodes de travail autonome.

26. C'est un point que nous avions plus spécialement développé dans une recherche antérieure à visée comparatiste (Bisault \& Le Bourgeois, 2006).

27. Recherche « analyse didactique des moments scolaires de découverte du monde " (2007-2010) financée par l'INRP en collaboration avec l'UMR STEF (ENS Cachan). 


\section{BIBLIOGRAPHIE}

Audigier, F., Crahay, M. \& Dolz, J. (2006). Curriculum, enseignement et pilotage. Bruxelles : De Boeck.

Bautier, E. (2006). Le rôle des pratiques des maîtres dans les difficultés scolaires des élèves. Recherche et formation, 51, 105-118.

Beaufils, D. \& Larcher, C. (1999). L'expérimental dans la classe. ASTER, 28, 3-8.

Berzin, C. (2000). Interactions de tutelle comme mode d'apprentissage à l'école? Psychologie Française, 45-3, 201-207.

Berzin, C \& Bisault, J. (2007). Activité et interactions inter-individuelles dans le cadre de l'apprentissage des sciences à l'école élémentaire : quelle contribution de la part du maître? Communication présentée au $3^{\text {eme }}$ colloque Constructivisme et Éducation. Genève. Septembre.

Bisault, J. (2005a). Faire des sciences pour apprendre à parler, lire et écrire ou parler, lire et écrire pour apprendre les sciences? In A. Giordan, J.-L. Martinand et D. Raichvarg (Eds.), Par les mots et par les choses, actes des XXVIIèmes Journées Internationales sur la Communication, l'Éducation et la Culture Scientifiques et Industrielles, [CDROM]. Paris : DIRES.

Bisault, J. (2005b). Langage, action et apprentissage en sciences à l'école maternelle. Spirale, 36, 123-138.

Bisault, J. (2005c). Le langage en sciences à l'école : quelles références et quels enjeux pour quelles pratiques scolaires? In A. Rouchier (Ed.), Actes du Colloque international « didactiques : quelles références épistémologiques ", [CDROM]. Bordeaux : IUFM d'Aquitaine.

Bisault, J. (2008). Interactions verbales, investigation expérimentale et conceptualisation en sciences à l'école primaire. Carrefours de l'éducation, 25, 17-31.

Bisault, J. \& Le Bourgeois, R. (2006). Les enjeux disciplinaires et transversaux de l'argumentation à l'école. L'exemple de l'histoire et des sciences. Les sciences de l'éducation. Pour l'ère nouvelle, 39, 101-139.

Bouchard, R. \& Traverso, V. (2006). Objets écrits et processus d'inscription entre planification et émergence. In M.-C. Guernier, V. Durand-Guerrier \& J.P. Sautot (dir.), Interactions verbales, didactiques et apprentissages : recueil, traitement et interprétation didactique des données langagières en contextes scolaires (pp 185-219). Presses universitaires de Franche-Comté.

Bronckart, J.P. (1996). Activité langagière, textes et discours : pour un interactionnisme socio-discursif. Lausanne - Paris : Delachaux et Niestlé.

Brousseau, G. (1998). Théorie des situations didactiques. Grenoble : La Pensée Sauvage.

Bruner, J.S. (1983). Le rôle de l'interaction de tutelle dans la résolution de problème. In J.S. Bruner (Ed.), Le développement de l'enfant : Savoir faire savoir dire (pp. 261-280). Paris : Presses Universitaires de France.

Chappet Paries, M. (2004). Comparaison de pratiques d'enseignants de mathématiques : relations entre discours des professeurs et activités potentielles des élèves. Recherches en didactique des mathématiques, 24 , 251-284.

Coquidé, M. (1998). Les pratiques expérimentales : propos d'enseignants et conceptions officielles. ASTER, 26, 109-132.

Fontaine, V., Vérin, A. \& Bisault, J. (2005). Le brouillard au cours moyen : une investigation empirique pour construire des connaissances. Grand N, 76, 83-98.

Dumas Carré, A. \& Weil Barais, A. Eds. (1998). Tutelle et médiation dans l'éducation scientifique. Berne : Peter Lang. Jaubert, M. \& Rebière, M. (2000). Observer l'activité langagière des élèves en sciences. ASTER 31. 173-196.

Larcher, C. (2003). Contribution à la table ronde « cadres théoriques autour de la modélisation ». In V. Albe, C. Orange \& L. Simonneaux (Eds.), Actes des $3^{\text {zemes }}$ rencontres scientifiques de l'ARDIST « Recherches en didactique des sciences et des techniques: Questions en débat » (pp. 305-308). Toulouse : ENFA.

Lebeaume, J. (2000). Jeux d'étiquettes, jeux de Kim, jeux de famille, puzzles ou devinettes à l'école. ASTER, $31,197-215$. 
Lebeaume, J. (2005). L'industrie humaine mise en mots pour l'école. In A. Giordan, J.-L. Martinand et D. Raichvarg (Eds.), Par les mots et par les choses, actes des XXVII İmes Journées Internationales sur la Communication, l'Éducation et la Culture Scientifiques et Industrielles, [CDROM]. Paris : DIRES.

Leplat, J. (1997). Regards sur l'activité en situation de travail, contribution à la psychologie ergonomique. Paris, PUF.

Martinand, J.-L. (2000). Rapport au savoir et modélisation en sciences. In A. Chabchoub (dir.), Rapports aux

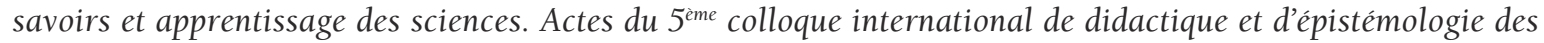
sciences, tome 1 (pp. 123-135). Tunis.

Martinand, J.-L. (2003). L'éducation technologique à l'école moyenne en France : problèmes de didactique curriculaire, Revue canadienne de l'enseignement des sciences, des mathématiques et des technologies, 3, 101-106.

Nonnon E. (2001). « La construction d'objets communs d'attention et de champs notionnels à travers l'activité partagée de description », in : Grandaty M et Turco G. (coord.). L'oral dans la classe : discours, métadiscours, interactions verbales et construction de savoirs à l'école primaire. Paris : INRP.

Orange, C., Fourneau, J.-C. \& Bourbigot, J.-P. (2001). Écrits de travail, débats scientifiques et problématisation à l'école élémentaire. ASTER, 33, 111-134.

Ross, A. (2000). Curriculum : construction and critique. London/New York : Falmer Press.

Sensevy, G. (2007). Des catégories pour décrire et comprendre l'action didactique. In G. Sensevy \& A. Mercier (Eds.), Agir ensemble: L'action didactique conjointe du professeur et des élèves (pp. 13-49). Rennes: Presses universitaires de Rennes (PUR).

Sensevy, G. \& Quilio, S. (2002). Les discours du professeur. Vers une pragmatique didactique. Revue Française de Pédagogie, 141, 47-56.

Sensevy, G. \& Turco, G. (2004). Interactions langagières, contenus de savoir et action du professeur : un débat sur le vivant en GS-CP. In Actes du colloque international «Faut-il parler pour apprendre? » [Cédérom]. Arras : IUFM Nord-Pas de Calais.

Schneuwly, B., Dolz, J., \& Roveaux, C. (2006). Le synopsis : un outil pour analyser les objets enseignés. In M.-J. Perrin-Glorian \& Y. Reuter (Eds.), Les méthodes de recherche en didactiques (pp 175-190). Villeneuve d'Ascq : Septentrion.

Schubauer-Leoni, M.-L., Leutenegger, F., Ligozat, F. \& Fluckiger, A. (2007). Un modèle de l'action conjointe professeur-élèves : les phénomènes didactiques qu'il peut/doit traiter. In G. Sensevy \& A. Mercier (Eds.), Agir ensemble: L'action didactique conjointe du professeur et des élèves (pp. 13-49). Rennes: Presses universitaires de Rennes (PUR).

Tiberghien, A., Buty, C. \& Le Maréchal, J.-F. (2003). La modélisation, axe prioritaire d'une approche théorique sur les relations entre apprentissage et enseignement. In V. Albe, C. Orange \& L. Simonneaux (Eds.), Actes des $3^{\text {emes }}$ rencontres scientifiques de l'ARDIST « Recherches en didactique des sciences et des techniques: Questions en débat » (pp. 309-314). Toulouse : ENFA.

Tiberghien, A., Malkoun, L., Buty, C., Souassy, N. \& Mortimer, E. (2007). Analyse des savoirs en jeu en classe de physique à différentes échelles de temps. In G. Sensevy \& A. Mercier (Eds.), Agir ensemble : L'action didactique conjointe du professeur et des élèves (pp. 93-122). Rennes : Presses universitaires de Rennes (PUR).

Venturini, P. (2007).Utilisation du rapport au savoir en didactique de la physique: un premier bilan. In P. Marquet, N. Hedjerassi, A. Jarlegan \& P. Remoussenard (Eds.), Actes du congrès AREF 2007 - Actualité de la Recherche en Éducation et en Formation [Cédérom]. Strasbourg : Université Louis Pasteur.

Vérin, A. (1995). Mettre par écrit ses idées pour les faire évoluer en sciences. Repères, 12, 21-36. 


\section{ANNEXE : RÉSULTATS DE L'ANALYSE DES ÉCHANGES SUR LES DIFFÉRENTS TYPES D'ACTIVITÉ EFFECTIVE}

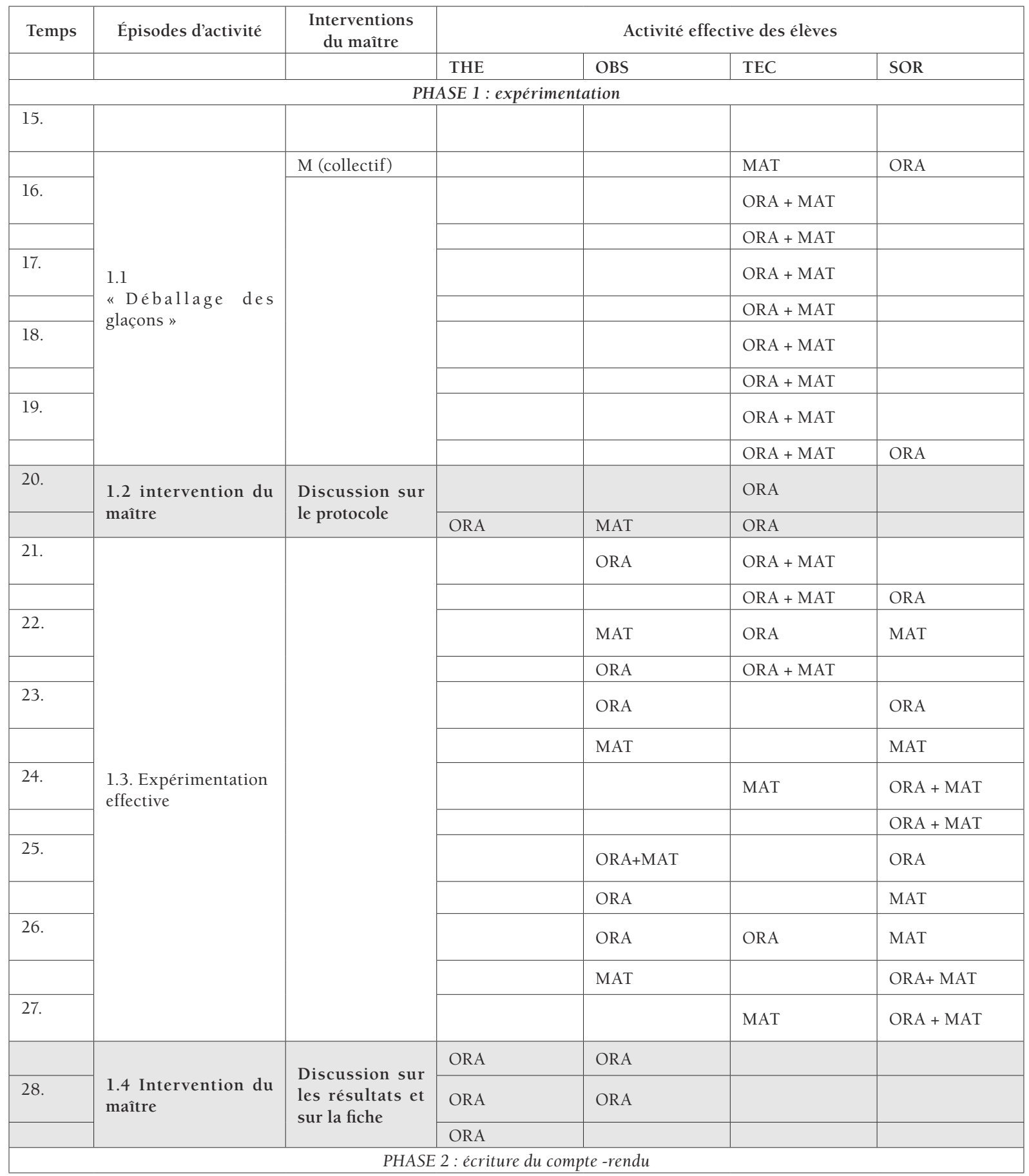




\begin{tabular}{|c|c|c|c|c|c|c|}
\hline \multirow[t]{2}{*}{29.} & \multirow{7}{*}{$\begin{array}{l}\text { 2.1. "Remplissage " } \\
\text { de la fiche }\end{array}$} & & & & ORA + ECR & \\
\hline & & & & MAT & ORA + ECR & \\
\hline \multirow[t]{2}{*}{30.} & & & & & ORA + ECR & MAT \\
\hline & & & & ORA + MAT & $\mathrm{ORA}+\mathrm{ECR}$ & \\
\hline \multirow[t]{2}{*}{31.} & & & & & ORA+ECR+MAT & \\
\hline & & & & & ORA + ECR & MAT \\
\hline \multirow[t]{2}{*}{32.} & & & & & ORA + ECR & \\
\hline & $\begin{array}{l}2.2 \text { Intervention } \mathrm{du} \\
\text { maître }\end{array}$ & $\begin{array}{l}\text { D iscussion } \\
\text { sur le contrat } \\
\text { d'écriture }\end{array}$ & & & ORA + ECR & \\
\hline \multirow[t]{2}{*}{33.} & \multirow{5}{*}{$\begin{array}{l}2.3 . \\
\text { Rédaction effective de } \\
\text { la fiche }\end{array}$} & & & & ORA + ECR & \\
\hline & & & & ORA + ECR & ORA & \\
\hline \multirow[t]{2}{*}{34.} & & & & $\mathrm{ORA}+\mathrm{ECR}$ & & \\
\hline & & & & $\mathrm{ORA}+\mathrm{ECR}$ & & \\
\hline \multirow[t]{2}{*}{35.} & & & & $\mathrm{ORA}+\mathrm{ECR}+\mathrm{MAT}$ & & \\
\hline & \multirow{7}{*}{$\begin{array}{l}2.4 \\
\text { Intervention du } \\
\text { maître }\end{array}$} & \multirow{7}{*}{$\begin{array}{l}\text { Discussion sur la } \\
\text { retranscription } \\
\text { des observations }\end{array}$} & & ORA & & \\
\hline \multirow[t]{2}{*}{36.} & & & & ORA + ECR & & \\
\hline & & & & ORA + ECR & ORA & \\
\hline \multirow[t]{2}{*}{37.} & & & & $\mathrm{ORA}+\mathrm{ECR}$ & ORA & \\
\hline & & & & ORA + ECR & & \\
\hline \multirow[t]{2}{*}{38.} & & & ORA & & & \\
\hline & & & & ORA & & \\
\hline \multirow[t]{2}{*}{39.} & \multirow{5}{*}{$\begin{array}{l}2.5 . \\
\text { Fin de la rédaction de } \\
\text { la fiche }\end{array}$} & & & ORA & & ORA \\
\hline & & & & $\mathrm{ORA}+\mathrm{ECR}+\mathrm{MAT}$ & & \\
\hline \multirow[t]{2}{*}{40.} & & & & ECR & & \\
\hline & & & & ORA+ECR & & \\
\hline 41. & & & & $\mathrm{ORA}+\mathrm{ECR}$ & ORA & ORA \\
\hline \multicolumn{3}{|c|}{ Total par type et modalité } & 5 ORA & $\begin{array}{l}23 \text { ORA } \\
12 \text { ECR } \\
9 \text { MAT }\end{array}$ & $\begin{array}{l}27 \text { ORA, } \\
9 \text { ECR, } \\
15 \text { MAT }\end{array}$ & $\begin{array}{l}11 \text { ORA, } \\
10 \text { MAT }\end{array}$ \\
\hline \multicolumn{3}{|c|}{ Total par type * } & 5 THE & 29OBS & 31 TEC & 17 SOR \\
\hline
\end{tabular}

* Le total par type est calculé pour le nombre d'occurrences de chaque type quelle que soit la modalité (une modalité double ou triple n'est comptée qu'une fois) : l'addition des occurrences par modalité pour chaque type est donc supérieure au total par type.

Chaque ligne correspond à une unité de temps de 30 s pendant laquelle l'activité des élèves a été caractérisée. Les lignes en grisé correspondent aux moments dirigés par le maître. Les interventions plus ponctuelles du maître ne sont pas représentées ici (on peut se reporter au document 7 pour une analyse de l'ensemble de ces interventions). 